\title{
Journal of Fluid Mechanics
}

http://journals.cambridge.org/FLM

Additional services for Journal of Fluid Mechanics:

Journal of

Email alerts: $\underline{\text { Click here }}$

Subscriptions: Click here

Commercial reprints: Click here

Terms of use : $\underline{\text { Click here }}$

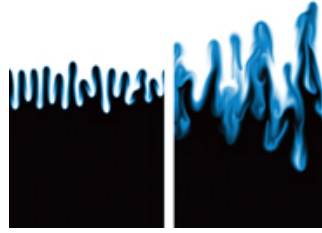

\section{Break-away separation for high turbulence intensity and large Reynolds number}

\author{
B. SCHEICHL, A. KLUWICK and F. T. SMITH
}

Journal of Fluid Mechanics / Volume 670 / March 2011, pp 260 - 300

DOI: 10.1017/S0022112010005306, Published online: 22 February 2011

Link to this article: http://journals.cambridge.org/abstract S0022112010005306

How to cite this article:

B. SCHEICHL, A. KLUWICK and F. T. SMITH (2011). Break-away separation for high turbulence intensity and large Reynolds number. Journal of Fluid Mechanics, 670, pp 260-300 doi:10.1017/ S0022112010005306

Request Permissions : $\underline{\text { Click here }}$ 


\title{
Break-away separation for high turbulence intensity and large Reynolds number
}

\author{
B. SCHEICHL $L^{1,2} \dagger$, A. KLUWICK ${ }^{1}$ AND F. T. SMITH $H^{3}$ \\ ${ }^{1}$ Institute of Fluid Mechanics and Heat Transfer, Vienna University of Technology, \\ Resselgasse 3/E322, A-1040 Vienna, Austria \\ ${ }^{2} \mathrm{AC}^{2} \mathrm{~T}$ Research $\mathrm{GmbH}$, Austrian Center of Competence for Tribology, Viktor Kaplan-Straße 2, \\ A-2700 Wiener Neustadt, Austria \\ ${ }^{3}$ Department of Mathematics, University College London, Gower Street, London WC1E 6BT, UK
}

(Received 3 March 2010; revised 10 August 2010; accepted 16 September 2010)

Massive flow separation from the surface of a plane bluff obstacle in an incompressible uniform stream is addressed theoretically for large values of the global Reynolds number $R e$. The analysis is motivated by a conclusion drawn from recent theoretical results which is corroborated by experimental findings but apparently contrasts with common reasoning: the attached boundary layer extending from the front stagnation point to the position of separation never attains a fully developed turbulent state, even for arbitrarily large Re. Consequently, the boundary layer exhibits a certain level of turbulence intensity that is linked with the separation process, governed by local viscous-inviscid interaction. Eventually, the latter mechanism is expected to be associated with rapid change of the separating shear layer towards a fully developed turbulent one. A self-consistent flow description in the vicinity of separation is derived, where the present study includes the predominantly turbulent region. We establish a criterion that acts to select the position of separation. The basic analysis here, which appears physically feasible and rational, is carried out without needing to resort to a specific turbulence closure.

Key words: boundary layer separation, boundary layers, turbulent flows

\section{Introduction}

Incompressible-flow separation past a blunt cylinder with an impervious inflexible smooth surface is of vital interest from an engineering point of view, where a reliable method is sought to predict the position of time-mean gross separation of the turbulent boundary layer. As Sandborn \& Liu (1968) stated at the very beginning of their experimental study, 'Turbulent boundary-layer separation is normally listed as one of the most important unsolved problems in fluid mechanics...', which represents quite a challenge. Considerable theoretical efforts however have not yet led to a fully self-consistent picture of the separation process even within the framework of a timemean description of the flow. Also, despite the rapid progress made in recent years in direct numerical simulation (DNS) and semi-direct numerical methods such as large-eddy and detached-eddy simulations, existing computational techniques do not master fully this great challenge by producing sufficiently accurate solutions of the 

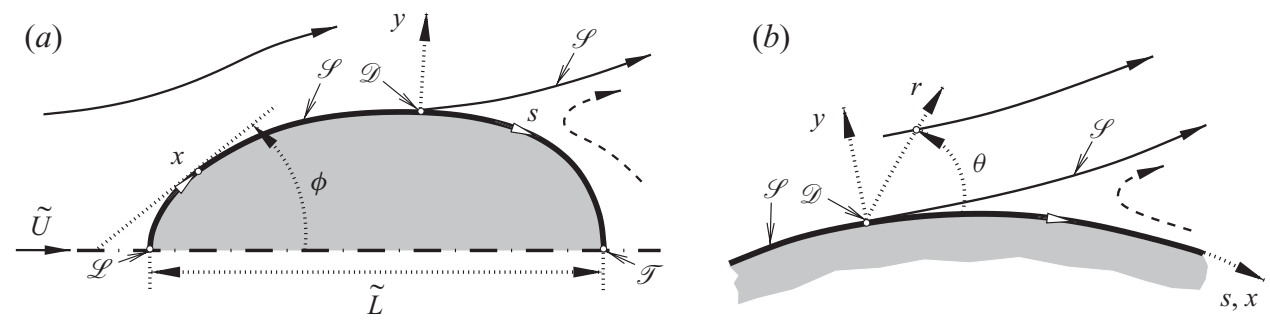

FIGURE 1. Sketch of flow situation, including streamlines of inviscid flow (arrows indicate flow direction, for labelling see text): — , free-stream region; -..-, slipstream; (a) global (here shown for a symmetric body) and $(b)$ local (near the position $\mathscr{D}$ of flow detachment).

unsteady Navier-Stokes equations in particular. This is largely because in practical applications, for instance in aerodynamics, the relevant Reynolds numbers must be large enough for the boundary and separated shear layer to exhibit rather high levels of turbulence intensity, and these Reynolds numbers are often too large to be dealt with adequately by numerical methods currently available.

The present study aims to shed some light on the intricate flow structure near separation with the goal of fostering future progress in modelling turbulent separating flows. It is desirable to gain deeper insight into two fundamental aspects which constitute the core problem: (i) the behaviour of the nominally two-dimensional steady flow in the vicinity of separation and (ii) how the local theory describing (i) fits into the global picture of the flow past the obstacle under consideration. In the following, all flow quantities are non-dimensional with respect to the speed $\tilde{U}$ of the unperturbed oncoming uniform flow, a typical body dimension $\tilde{L}$ (see figure $1 a$ ), and the (constant) fluid density. Furthermore, the global Reynolds number $R e$ takes on arbitrarily large values,

$$
R e=\tilde{U} \tilde{L} / \tilde{v} \gg 1,
$$

where the constant $\tilde{v}$ is the kinematic viscosity of the fluid. Analytical methods such as matched asymptotic expansions then provide an appropriate means to establish a rational theory on the basis of the time- or, equivalently, Reynolds-averaged NavierStokes (RANS) equations, i.e. the Reynolds equations.

In laminar flow (however inadequate that presumption may be on physical grounds), the triple-deck structure provides a rational description of break-away separation for (1.1), at least locally (see Sychev 1972; Smith 1977). For an overview of the laminar bluff-body problem, including the controversy concerning the validity and preference of a particular model of the large-scale separated flow, see Smith $(1979,1985,1986)$, Chernyshenko (1988) and Sychev et al. (1998) for instance; the currently accepted solution was put forward by Chernyshenko (1988), and an overview of the whole subject is in Sychev et al. (1998). In turbulent flow, the current state of asymptotic theory for the fully developed turbulent case is described by Kluwick \& Scheichl (2009). A local description of the separation process was attempted systematically in interesting original works by Sychev \& Sychev (1980), Sychev (1983) and Sychev (1987), Melnik (1989). For further references and a discussion of these enthralling and illuminating attempts, see Neish \& Smith (1992) and Scheichl \& Kluwick (2008a). In a recent development (Scheichl, Kluwick \& Alletto 2008; Scheichl \& Kluwick $2008 b$ ), a self-consistent flow structure matching the boundary layer region with the small region of pronounced laminar-turbulent transition near the leading edge of the obstacle is found to agree with the classical picture of a two-tiered turbulent boundary 
layer. It consists of, first, the fully turbulent outer main region comprising most of the boundary layer, which exhibits a small defect of the streamwise velocity component with respect to its value imposed by the external potential flow and has Reynolds stresses dominating over the viscous shear stress, and, second, the viscous wall layer, where the turbulent shear stress and its molecular counterpart are of comparable magnitude. In the present study that classical structure is adjusted in order to also account for underdeveloped turbulence (following the measurements of Schewe 2001 and suggestions of Scheichl et al. 2008 and Scheichl \& Kluwick $2008 b$ below), i.e. for a boundary layer characterised by a level of turbulence intensity below that of a fully developed turbulent boundary layer flow. In the present investigation, this concept is restricted to sufficiently high turbulence intensities, so that the turbulent boundary layer already exhibits the aforementioned typical two-tiered structure. More specifically, here the notion of slightly underdeveloped turbulence turns out to be crucial.

This type of 'transitional' boundary layer flow was originally proposed by Neish \& Smith (1992). In its form adopted here, two asymptotically small perturbation parameters are employed: a measure for the velocity defect, $\epsilon$, and a further measure, $\sigma$, for the boundary layer thickness, denoted by $\delta$. Hence, the quantity

$$
I_{t}=\alpha \epsilon^{2}, \quad \text { with } \quad \alpha=\sigma / \epsilon,
$$

measures the magnitude of the correlation of the turbulent velocity fluctuations, i.e. of the Reynolds stresses or the turbulence intensity, in the shear layer. We deal with slightly underdeveloped turbulent flow if both of the following relationships hold:

$$
\begin{gathered}
\epsilon \ln R e=O(1), \\
\alpha \ll 1 .
\end{gathered}
$$

This eventually assumes its fully developed form if both $\sigma$ and $\epsilon$ are $O(1 / \ln R e)$, i.e. then $\alpha=O(1)$. Both the ranges (1.3) control the turbulence intensity levels, where (1.3b) alone emerges to characterise underdeveloped turbulence. The process of laminarturbulent transition provides a source (Scheichl et al. 2008; Scheichl \& Kluwick 2008b) of delaying the boundary layer flow from becoming a fully developed turbulent flow, so that we speak of a laminar-turbulent boundary layer in the following. Selfconsistency of the slightly underdeveloped-flow scaling is confirmed by considering the local asymptotic structure of the boundary layer close to separation: free-stream convection penetrates through the outer layer to the top of the wall layer, which is exponentially thin compared to the former in the classical (fully developed) case. Then the pressure feedback exerted by the induced inviscid region that feels the local displacement of the flow adjacent to the surface is too weak to alter this near-wall flow to leading order and avoid the formation of a Goldstein singularity (cf. Rothmayer \& Smith 1998; Sychev et al. 1998). Thus, a sound formulation of this locally strong viscous-inviscid interaction process requires the von Kármán number $\delta^{+}$(namely the ratio of the inner- and outer-layer thicknesses) to vary essentially algebraically with $1 / \delta$ rather than exponentially as in the classical case. In other words, $\delta$ is found to depend predominantly algebraically on $R e$ rather than logarithmically, associated with the classical scaling. Moreover, a detailed analysis of a turbulent boundary layer evolving from the leading edge towards the location of separation (Scheichl \& Kluwick 2008b) indicates that the first situation leads to a specific (distinguished) double limit $\epsilon \rightarrow 0, \alpha \rightarrow 0$, such that the associated dependence of $\alpha$ on Re determines the ultimate value of the turbulence-intensity level $I_{t}$ possible. 
Altogether, turbulent separation is found to be associated with a quite complex interplay of a so-called inner and an outer mechanism of local viscous-inviscid interaction. In this paper we concentrate on both mechanisms; however, particular details of the latter and its interplay with the inner interaction will be addressed more elaborately in a later study. The outer interaction is of paramount importance for the understanding of the drastic change of the flow in the wall layer as it undergoes separation, itself governed by the inner interaction process. The inner interaction process gives rise to a novel internal triple-deck structure which is located at the base of the boundary layer and which eventually fixes the value of $I_{t}$.

The concept of slightly underdeveloped turbulence adopted in the present analysis is novel and somewhat unconventional but is supported by the oil-flow measurements carried out by Schewe (2001), as discussed by Scheichl et al. (2008) and Scheichl \& Kluwick (2008b). Also, Sandberg \& Sandham (2008) conclude, albeit with some reservation, from the results of their DNS study that the turbulent flow close to the trailing edge of a flat plate aligned in a uniform stream exhibits characteristics which point to the well-known triple-deck structure as in laminar flow (see Rothmayer \& Smith 1998; Sychev et al. 1998). Although not directly concerned with separation from a smooth surface, this finding is of particular interest: it is demonstrated below how the asymptotic representation of the flow near separation in the regime of the outer interaction is closely related to that occurring in the turbulent flow past the trailing edge of a flat plate at angle of attack as considered first by Melnik \& Chow (1975). The differences are mainly due to different near-wall boundary conditions downstream of separation.

This paper is organised as follows. After the problem description on the basis of the Reynolds equations in $\S 2$, an outline for the external and boundary layer flows is given in $\S 3$, concluded by the rationale supporting the existence of an underdeveloped turbulent boundary layer flow and the definition of the slightly underdeveloped case. The results presented lead to a complete flow picture and a thorough investigation of the inner and (less elaborately) the outer interaction process as in $\S 5$. The solutions of the impressed potential flow as well as the boundary layer equations that govern the outer small-defect portion of the oncoming turbulent flow enter the interactiveflow description in the form of certain parameters and require both analytical and numerical treatments: they are discussed separately in $\S 4$. Finally, the implications of the description of separation here are highlighted in $\S 6$. Also, the assumptions made in the course of the analysis having the most important repercussions on its progress are summarised there.

\section{Problem formulation}

As a starting point, we formulate the basic equations governing the time-mean flow past the cylindrical body under consideration. Here and in $\S 3$ we tacitly refer to figure 1 whenever necessary.

Let $x, y, u, v, \psi, p$ denote natural coordinates along and perpendicular to the upper part of the perfectly smooth body surface, respectively, having the origin in the point $\mathscr{L}$ defining the leading edge of the cylinder, the velocity components in the $x$ and $y$-directions, the streamfunction, and the pressure. Furthermore, $\phi(x)$ denotes the angle of inclination of the surface contour at a distance $x$ measured anticlockwise from the direction of the oncoming parallel flow. Then, the value of the surface curvature

$$
x(x)=-\mathrm{d} \phi / \mathrm{d} x
$$


is positive/negative for a convex/concave part of the contour. In turn, the continuity equation is satisfied identically with $u=\partial_{y} \psi, h v=-\partial_{x} \psi$, where $h=1+\chi y$. Hence, the Reynolds equations consist of the momentum equations for the $x$ - and $y$-directions (see e.g. Schlichting \& Gersten 2003, p. 81),

$$
\begin{aligned}
h^{2} D_{t} \partial_{y} \psi-\chi\left(\partial_{x} \psi\right)\left(\partial_{y} \psi\right) & =-h \partial_{x} p-h \partial_{x}\left\langle u^{\prime 2}\right\rangle-\partial_{y}\left(h^{2}\left\langle u^{\prime} v^{\prime}\right\rangle\right)+v h^{2} \partial_{y} \nabla^{2} \psi \\
-h D_{t}\left(h^{-1} \partial_{x} \psi\right)-\chi\left(\partial_{y} \psi\right)^{2} & =-h \partial_{y} p-\partial_{y}\left(h\left\langle v^{\prime 2}\right\rangle\right)-\partial_{x}\left\langle u^{\prime} v^{\prime}\right\rangle+\varkappa\left\langle u^{\prime 2}\right\rangle-v \partial_{x} \nabla^{2} \psi
\end{aligned}
$$

where $v=R e^{-1}$ (for the sake of brevity) and the total derivative and the Laplacian are

$$
D_{t}=h^{-1}\left[\partial_{y} \psi \partial_{x}-\partial_{x} \psi \partial_{y}\right], \quad \nabla^{2}=h^{-1}\left[\partial_{x}\left(h^{-1} \partial_{x}\right)+\partial_{y}\left(h \partial_{y}\right)\right] .
$$

Also, $u^{\prime}, v^{\prime}$ denote the turbulent fluctuations of the $x$ - and $y$-velocity components, respectively. Thus, the components of the Reynolds stress tensor are identified by $\langle\cdot\rangle$. Equations (2.2) are supplemented with the usual no-slip and no-penetration boundary conditions at the solid cylinder surface and the requirement for a uniform parallel flow infinitely remote from the body, such that for

$$
\begin{aligned}
y=0: & u=v=u^{\prime}=v^{\prime}=0, \\
y \rightarrow \infty: & {\left[u, v, u^{\prime}, v^{\prime}, p\right] \rightarrow[\cos \phi,-\sin \phi, 0,0,0] . }
\end{aligned}
$$

Here $\phi$ is the slope angle introduced earlier.

Furthermore, in the following we tacitly assume that all components of the Reynolds stress tensor have the same order of magnitude under the assumption (1.1) (hypothesis of locally isotropic turbulence; cf. Kolmogorov 1961 and the references therein).

We subsequently aim at investigating the flow in the vicinity of separation in the regime (1.1) on the basis of (2.2)-(2.4). To this end, we start with considerations regarding the overall asymptotic picture of the (non-interactive) flow as put forward by Scheichl et al. (2008) and Scheichl \& Kluwick (2008b). This is the subject of $\S 3$.

\section{Overall background and preliminary results}

The structure of the flow on the scale of body dimensions under the assumption (1.1) represents a decisive but delicate issue still under debate. Nonetheless, its rather few salient properties underlying the present analysis seem plausible.

\subsection{Does Euler flow provide a feasible asymptotic state for large values of Re?}

As will be argued in $\S 3.4 .1$, the attached boundary layer emanating from $\mathscr{L}$ exhibits underdeveloped turbulence and thus typically shrinks towards the line $y=0$ as $R e$ increases. On the other hand, it is a widely accepted fact that a free turbulent shear layer is a fully developed and 'thick' one insofar as its thickness is essentially independent of $R e$ and measures the turbulence intensity concentrated in it. However, from an empirical point of view, such a shear layer can still be regarded as relatively slender though, as was first put in a formal asymptotic concept successfully by Schneider (1991); for boundary layers see Melnik (1989) and Scheichl \& Kluwick (2007b). Hence, the existence of separated flow here does not restrain us from regarding the Reynolds stresses as negligibly small entirely within an extent of a typical body dimension from the body surface, so that $(2.2 a)$ and $(2.2 b)$ reduce to the Euler equations in the present regime. Therefore, the question posed at the beginning of this section is answered in the affirmative, even if tentatively.

We concede, of course, that the real behaviour of the flow may be different due to the effect of the free shear layers, but, hopefully, not too different from the model 
proposed here, so that it can be adapted in a self-consistent manner. We are also aware that the picture of the global flow remains incomplete as long as transition of the shear layer just separated to a fully developed shear layer is accepted without being understood in detail. An interesting step in this direction was made recently by Sychev (2010).

The flow behaviour considered here is then accounted for by the leading-order term of the expansion

$$
[\psi, u, v, p] \sim\left[\psi_{0}, u_{0}, v_{0}, p_{0}\right](x, y ; k)+O(\epsilon \sigma), \quad y=O(1),
$$

which is suggested by the form of the small-defect structure of the boundary layer as addressed briefly in $\S 1$. The non-negative parameter $k$ shall account for the initially unknown location $x=x_{\mathscr{D}}(k)$ of the point $\mathscr{D}$ where the free streamline, on which $\psi_{0}=0$ by definition and here denoted by $\mathscr{S}$, detaches tangentially from the body surface. A precise definition of $k$ that involves the structure of the flow near $\mathscr{D}$, is given in the course of its further discussion (see $\S 3.3$ ). Next, we recapitulate the essential topological properties of that Euler flow which, in view of (1.1), is formally assumed in the limit of arbitrarily large values of $R e$.

\subsection{Global picture of inviscid flow}

The inviscid free-stream flow, characterised by $y \geqslant y_{\mathscr{S}}$, is irrotational. The potential flows past a cylinder that are parametrised by $k$ and leave a (semi-infinitely) open cavity, i.e. a dead-water zone where $p_{0} \equiv 0$ according to the far-field conditions (2.4), downstream of the body are usually referred to as Helmholtz-Kirchhoff (H-K) flows; for an extensive treatment, see e.g. the textbook by Gurevich (1979). It is self-evident that in this case, (2.4) only holds outside the cavity. In contrast to the more general approach proposed subsequently but according to what is conventionally suggested in previous related studies, it should be mentioned that Sychev et al. (1998) (also cf. the references therein) and Schlichting \& Gersten (2003) consider this type of a potential flow as the only physically realistic flow that is representative in the case (1.1), in agreement with the prerequisites of the original analysis of laminar separation by Sychev (1972), at least as far as steady flows are concerned.

In the following, we relax the assumption that in the inviscid-flow limit, formally expressed as $R e^{-1}=0$, the slipstream of the body degenerates into an open cavity: it is equivalently reasonable from the viewpoint of first principles that the terminal asymptotic picture of the flow about the cylinder exhibits a closed region that forms either a dead-water cavity (cf. Eppler 1954; Gurevich 1979) or even an inviscid recirculating flow with (negative) vorticity, hereafter denoted by $\omega_{0}$, behind the body. This region then is assumed to have an extent comparable to a characteristic dimension of the body and thus to lie within a distance $y=O(1)$ from the trailing edge, denoted by $\mathscr{T}$. Under the assumption of a dead-water region confined by $\mathscr{S}$, the first possibility applies when $\mathscr{D}$ is sufficiently close to $\mathscr{T}$. Then, the uniform $k$-dependent value of $p_{0}$, typical for the (cusp-shaped) dead-water zone, is positive. Note, however, that this patently contrasts with experimental findings as these support the existence of a sub-pressure cavity (cf. Zdravkovich 1997), even for $R e \doteq 1.782 \times 10^{7}$ for a circular cylinder and $R e$ formed with its diameter (Jones, Cincotta \& Walker 1969). The second possibility is associated with a rich variety of complex flow pictures and comprises the first situation by including the trivial case $\omega_{0} \equiv 0(y \geqslant 0)$. Because of the inherent impact of turbulent unsteadiness on the flow even for arbitrarily large values of $R e$, for $\omega_{0} \neq \equiv 0$ the (two) counter-rotating eddies are not necessarily of the celebrated Prandtl-Batchelor type, where each eddy is identified by a uniform value 

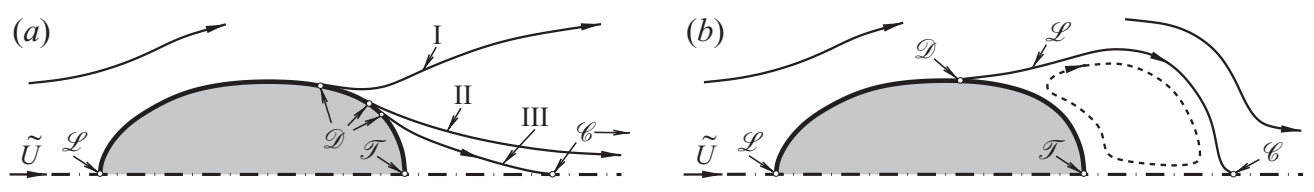

FiguRE 2. Sketch of inviscid (here symmetric) bluff-body flows (for labelling see text and figure 1): $\mathscr{S}$ separating $(a)$ a cavity and $(b)$ a vortex flow (here assumed to form a cusp near $\mathscr{C}$ ).

of $\omega_{0}$ (Batchelor 1956a,b): the latter situation only applies if the unsteady terms in the Navier-Stokes equations tend to zero faster than the viscous terms for increasing values of Re. In passing, we note that critical overviews on such categories of inviscid flows, exhibiting different forms of closed wakes, are put forward by Wu (1972), Zdravkovich (1997) and Sychev et al. (1998). Both scenarios discussed so far are displayed in figure 2 , where $\mathscr{C}$ denotes the point confining either the cavity or the wake flow on the centreline. In the first scenario, the free streamlines $\mathscr{S}$ indicated by I, II and III refer to the cases $k<k_{c}$ (H-K flow), $k=k_{c}$ ( $\mathscr{C}$ infinitely remote) and $k>k_{c}$ ( $\mathscr{C}$ finitely remote), respectively, for some critical value $k_{c}$ depending on the body geometry.

We now focus briefly on the specific asymptotic structure of the external Euler flow and the initially attached boundary layer, by both taking up a global point of view and scrutinising the vicinity of the point of separation $\mathscr{D}$.

\subsection{Inviscid free-streamline theory reappraised}

The reasoning outlined so far in $\S 3$ implies that the Euler flow around the cylinder is sought in the class of flows that are irrotational far upstream (and downstream), exhibit free streamlines and, as a consequence, are essentially parametrised by the parameter $k$. A careful investigation of the inviscid flow near $\mathscr{D}$ provides a first step towards the understanding of the separation process.

By introducing a local coordinate

$$
s=x-x_{\mathscr{D}}(k),
$$

let $y=y_{\mathscr{S}}(s ; k)$ describe the position of $\mathscr{S}$, so that $y_{\mathscr{S}}=0$ for $s \leqslant 0$ (upstream of $\mathscr{D}$ ) and $y_{\mathscr{S}}>0$ for $s>0$ (downstream of $\mathscr{D}$ ). That is, $\mathscr{S}$ separates the irrotational freestream flow $\left(y \geqslant y_{\mathscr{S}}\right)$ from the slipstream $\left(s>0,0 \leqslant y<y_{\mathscr{S}}\right)$. Also, we introduce the speed $U_{\mathscr{S}}(x ; k)$ of the inviscid flow on $\mathscr{S}$. The flow in $\mathscr{D}$ is then characterised by the as yet unknown values $u_{0 \mathscr{P}}=U_{\mathscr{S}}\left(x_{\mathscr{D}} ; k\right)=u_{0}\left(x_{\mathscr{O}}, 0 ; k\right), p_{0 \mathscr{P}}=p_{0}\left(x_{\mathscr{D}}, 0 ; k\right)$, and the local surface curvature $x_{\mathscr{D}}=\chi\left(x_{\mathscr{O}}\right)$ according to (2.1). For $s>0$, in the following the subscripts + and - distinguish the different values of $U_{\mathscr{S}}$ for $y-y_{\mathscr{S}}=0_{+}$and $y-y_{\mathscr{S}}=0_{-}$, respectively. In the light of the central investigation, it seems expedient to demonstrate concisely how one can determine the asymptotic representations of $\psi_{0}$, $p_{0}$ (see (3.1)), $U_{\mathscr{S}}$ and $y_{\mathscr{S}}$ in the vicinity of $\mathscr{D}$ term by term iteratively (in principle with arbitrary accuracy) by prescribing $\omega_{0}\left(\psi_{0} ; k\right)$. As a matter of fact, the specific form of $\omega_{0}\left(\psi_{0} ; k\right)$ is expected to be known once the self-consistent asymptotic structure of the large-scale separated flow under the assumption (1.1) is understood satisfactorily well.

Here we list the results of the local analysis of flow detachment possessing ramifications for the remaining study, where the details are left to Appendix A. At first, we obtain

$$
\psi_{0} / U_{\mathscr{S}} \sim y-x y^{2} / 2+O\left(y^{3}\right), \quad s \leqslant 0, \quad y \rightarrow 0 .
$$


By setting $p_{\mathscr{S}}(x ; k)=p_{0}$ on $\mathscr{S}$, we have

$$
p_{\mathscr{S}}+U_{\mathscr{S}}^{2} / 2=1 / 2, \quad \psi_{0} \geqslant 0,
$$

and $p_{0 \mathscr{D}}=\left(1-u_{0 \mathscr{D}}^{2}\right) / 2$ for the pressure at separation. Polar coordinates

$$
r=\sqrt{s^{2}+y^{2}}, \quad \theta=\arctan (y / s) \quad(\pi \geqslant \theta \geqslant 0)
$$

come into operation as they facilitate the resultant expansion of $\psi_{0}$ in suitable form,

$$
\begin{aligned}
& \psi_{0} / u_{0 \mathscr{D}} \sim r \sin \theta+\psi_{0,3 / 2}(r, \theta ; k)+\psi_{02}(r, \theta ; k)+O\left(r^{5 / 2}\right), \quad \pi \geqslant \theta>0, \quad r \rightarrow 0 \\
& \psi_{0,3 / 2}=-\frac{4 k}{3} r^{3 / 2} \cos (3 \theta / 2), \quad \psi_{02}=-r^{2}\left\{\frac{5 k^{2}}{3} \sin (2 \theta)+\frac{\varkappa_{\mathscr{D}}}{4}[1-\cos (2 \theta)]\right\} .
\end{aligned}
$$

Finally, we obtain

$$
\begin{aligned}
U_{\mathscr{S}} \sim b(k) x+O\left(x^{2}\right), \quad b(k)>0, & x \rightarrow 0_{+}, \\
U_{\mathscr{S}} / u_{0 \mathscr{D}} \sim 1+2 k(-s)^{1 / 2}+\left(10 k^{2} / 3\right)(-s)+O\left[(-s)^{3 / 2}\right], & s \rightarrow 0_{-},
\end{aligned}
$$

and in turn, with the aid of (3.4),

$$
p_{\mathscr{S}} / u_{0 \mathscr{D}}^{2} \sim\left(u_{0 \mathscr{D}}^{-2}-1\right) / 2-2 k(-s)^{1 / 2}-\left(16 k^{2} / 3\right)(-s)+O\left[(-s)^{3 / 2}\right], \quad s \rightarrow 0_{-},
$$

and

$$
\begin{aligned}
U_{\mathscr{S}_{+}} / u_{0 \mathscr{D}} \sim 1-\left(\omega_{0 \mathscr{D}} / u_{0 \mathscr{D}}^{2}\right)(2 k / 3) s^{3 / 2}+O\left(s^{5 / 2}\right), & s \rightarrow 0_{+}, \\
y_{\mathscr{S}} \sim(4 k / 3) s^{3 / 2}+O\left(s^{5 / 2}\right), & s \rightarrow 0_{+} .
\end{aligned}
$$

From $(3.9 b)$, it immediately follows that indeed $k \geqslant 0$ for geometrical reasons. The region of non-uniformity along $\mathscr{S}$ mentioned at the end of item (i) in Appendix A emerges for $y=O\left(s^{3 / 2}\right)$ and represents the early-stage separated shear layer in the inviscid limit. In the above added form $(3.7 a)$ of $U_{\mathscr{S}}$ near $\mathscr{L}$ the coefficient $b$ reflects the local behaviour of the stagnant potential flow. Expansions (3.6)-(3.9) represent a summary of the results that are essential for the further analysis. We remark that they are uniformly valid in the regime $k \geqslant 0$.

The vorticity $\omega_{0}$ is found to enter the description of the potential flow only in the truncated terms in $(3.6 a),(3.7 b)$ and (3.8), which are not considered here. The singular behaviour of $\psi_{0}$ near $\mathscr{D}$ accounted for by the second contributions to these expansions and in (3.9) reflects the well-known Brillouin-Villat (B-V) singularity (cf. Gurevich 1979), which we have retrieved without restricting to the conventional assumption that the free streamline $\mathscr{S}$ confines a dead-water region $\left(\omega_{0} \equiv 0\right)$. Accordingly, the results (3.6), important in the following, have been derived directly rather than from the global solution of the potential flow, which in the existing literature is usually obtained in a distinctly more cumbersome manner by means of conformal mapping. A short survey on this procedure is presented in Appendix B.

The strength of the B-V singularity is measured by the flow parameter $k$, which virtually controls the magnitude of the unbounded adverse pressure gradient immediately upstream of $\mathscr{D}$, given by (3.8) in the form $\mathrm{d} p_{\mathscr{S}} / \mathrm{d} x \sim k(-s)^{-1 / 2}+O(1)$, as $s \rightarrow 0_{-}$. In this study, we deal with $k=O(1)$, which contrasts with the description of laminar break-away separation where $k=O\left(R e^{-1 / 16}\right)$ (cf. Sychev 1972; Smith 1977; Sychev et al. 1998). However, it is well-accepted that turbulent boundary layers are less prone to separate than laminar ones, which substantiates the present approach, referred to as 'non-smooth' separation by Neish \& Smith (1992), in allusion to the singular behaviour of the curvature of $\mathscr{S}$ given by $(3.9 \mathrm{~b})$. That is, we anticipate 
that one cannot determine the actual value of $k$ and, in turn, the position of $\mathscr{D}$ without having gained a considerably deeper insight in the interplay of the boundary layer and the large-scale separated-flow structure. In case of $\omega_{0} \equiv 0$ and for a convex cylinder $x_{\mathscr{D}}$ is shifted towards $\mathscr{T}$ for increasing values of $k$, accompanied by decreasing values of $u_{0 \mathscr{D}}$. It should be mentioned that the assumption $k \gg 1$ refers to separation asymptotically close to $\mathscr{T}$ where $u_{0 \mathscr{D}}$ becomes asymptotically small (see figure $2 a$ ). This limiting scenario has been first addressed by Neish \& Smith (1992) and critically reviewed by Scheichl \& Kluwick (2008a), at least for the case of a fully developed turbulent boundary layer immersed in fully attached potential flow, here recovered in the formal limit $k^{-1}=0$ under the premise (1.1).

\subsection{Incident attached boundary layer}

The attached portion of the boundary layer forming in the regime (1.1) is 'trapped' as it stretches approximately from $\mathscr{L}$ to $\mathscr{D}$ over a finite distance, regardless of the actual value of $R e$. This characteristic of bluff-body flows is apparently strongly linked to the effective magnitude of the asymptotic parameters $\epsilon, \sigma$ and $\alpha$, introduced in $\S 1$, and the associated level of turbulence intensity $I_{t}$, defined in (1.2), that prevails in the entire boundary layer. We start with an outline of the generation of the turbulent boundary layer close to $\mathscr{L}$. This specific picture of short-scale transition is, in turn, found to promote remarkably the assumption of underdeveloped turbulence. Finally, this state of flow is identified by $(1.3 b)$, which results from the order-of-magnitude analysis of $\epsilon$ and $\sigma$ performed in $\S 3.4 .3$.

\subsubsection{Onset of turbulence}

In the setting of the original formulation of boundary layer turbulence by Neish \& Smith (1992), which also covers underdeveloped turbulence, the Reynolds shear stress in the boundary layer is written as

$$
-\left\langle u^{\prime} v^{\prime}\right\rangle=T R e^{-1 / 2} \bar{\Sigma}(x, \bar{y} ; k, T), \quad \bar{y}=y R e^{1 / 2} .
$$

Here $T$ and $\bar{\Sigma}$ denote, respectively, the non-negative constant so-called turbulenceintensity gauge factor that serves to quantify the magnitude of the Reynolds shear stress, and a shape function (that has to be modelled). Owing to this scaling, the term $T \partial_{\bar{y}} \bar{\Sigma}$ adds to the conventional Prandtl-type boundary layer equations (formed with $x, \bar{y}$ used as independent variables). Their solutions are then parametrised by $T$ (aside from $k$ ). They describe a purely laminar flow for $T=0$, whereas the subsequent interesting case of highly developed turbulence is indicated by $T \gg 1$. It will become evident from the subsequent investigation that the latter case is preferably described by virtue of the formalism outlined in $\S 1$, i.e. in terms of the two parameters $\epsilon$ and $\sigma$, forming $I_{t}$, and their asymptotic dependencies both on $T$ and $R e$, instead of $T$ and $R e$.

The analysis by Scheichl et al. (2008) and Scheichl \& Kluwick (2008b) suggests that for arbitrarily large values of $R e$ the boundary layer evolves from the well-understood stagnant laminar flow (cf. Schlichting \& Gersten 2003) taking place in a small vicinity of $\mathscr{L}$ with an extent of $R e^{-1 / 2}$ and undergoes rapid laminar-turbulent transition in a relatively small adjacent region where the flow is still of boundary layer type. The idea that $U_{\mathscr{S}}$ provides the only reference velocity of that flow suggests the scaling relation $\bar{\Sigma}=O\left(U_{\mathscr{S}}^{2}\right)$. An inspection of magnitude analysis of $(2.2 a)$ in connection with (3.7a) yields then the estimates $x=O\left(T^{-1}\right), \bar{y}=O(1)$ for this region of transition, which in turn implies

$$
T R e^{-1 / 2} \ll 1 .
$$


Furthermore, we recall that in the regime (1.1) the turbulent dynamics is more or less constricted to a relatively thin boundary layer present for $x=O(1)$. Under this basic supposition, matching this developed turbulent boundary layer flow with that early-stage turbulent flow is found to require a small streamwise velocity deficit with respect to $U_{\mathscr{S}}$ in the bulk of the former, i.e. $1-u / U_{\mathscr{S}} \ll 1$. However, the scalings of the Reynolds stresses and in turn of the boundary layer thickness $\delta$ first remain unknown. To proceed with the scaling of the flow, the most simple strategy, motivated by physical reasoning and consistent with the considerations above leading to (3.11), is to assume that both regions have in common that the turbulent motion is governed by a single velocity scale: this is initially represented by $U_{\mathscr{S}}$, which is superseded by a turbulent reference velocity $u_{t}(x ; k, R e)$, when $x=O(1)$, so that in this flow region

$$
1-u / U_{\mathscr{S}}=O(\gamma), \quad \bar{\Sigma} / U_{\mathscr{S}}^{2}=O\left(\gamma^{2}\right), \quad \text { with } \quad \gamma=u_{t} / U_{\mathscr{S}} \sim \epsilon .
$$

The advanced (far-downstream) stage of the transition process characterised by an intermediate limit of the form $T^{-1} \ll x \ll 1$ is associated with a correspondingly pronounced reduction of the streamwise velocity defect in the main region of the boundary layer. Simultaneously, the value of the turbulence-intensity level $I_{t}$ increases and eventually reaches its maximum for $x=O(1)$. There the velocity defect is $O(\epsilon)$ finally, which motivates us to stipulate asymptotic proportionality between $\epsilon$ and $\gamma$ in (3.12). Specifically, it will become evident below how the definition of $\epsilon$ is traced back to this relation, without any loss of generality.

The turbulent boundary layer is driven by $U_{\mathscr{S}}$, known from the solution for $\psi_{0}$, from $\mathscr{L}$ towards $\mathscr{D}$. Its behaviour near these critical points is essentially determined by the local expansions (3.7). We initially reconsider the relevant conclusions inferred from the first-order description of (underdeveloped) turbulent boundary layer flow.

\subsubsection{First-order theory}

As brought out by e.g. Schlichting \& Gersten (2003), turbulent shear flows are intrinsically tied to a rather pronounced change from the external almost irrotational to the fully turbulent flow inside the boundary layer. In a rigorous asymptotic framework, this is accounted for by the outermost tier of the boundary layer that is extremely thin relative to its main region. It is formed at the aforementioned final stage of the transition process and accompanied by an increase of the boundary layer thickness proportional to $x$ (which reflects the vanishing effect of the viscous shear stress and thus the lack of a typical boundary layer length scale in the regime $\left.T^{-1} \ll x \ll 1\right)$. Since in the present context the specific properties of that overlayer do not play an important role, it can be approximated with sufficient accuracy by the sharp line $y=\delta$, with $\delta=O(\sigma)$. Then, the distinct outer edge of the boundary layer and its thickness $\delta$ are well-defined. Therefore, a patching of the flow quantities is required at $y=\delta$, specifically for $u$ and the boundary layer approximation $-\partial_{y} u$ of the vorticity, such that $u$ is smooth there. Taking up the more computational point of view, we accomplish this by employing a mixing-length-based closure of the Reynolds shear stress, notwithstanding the alternative (rather more conventional) eddy-viscosity-based approach in favour of claiming a diffusive decay of vorticity. This is aligned with the usual technique of matching as known from laminar shear flows and adopted by e.g. Neish \& Smith (1988, 1992).

As an even more important finding, (2.2) and (3.3) imply $p \sim p_{\mathscr{S}}+U_{\mathscr{S}}^{2} x y+O\left(\epsilon^{2}, \alpha^{2} \epsilon^{2}\right)$ throughout the turbulent boundary layer. In the outer small-defect region, (2.2a) then reduces to the differential form of (3.4), $\mathrm{d} p_{\mathscr{S}} / \mathrm{d} x=-U_{\mathscr{S}} \mathrm{d} U_{\mathscr{S}} / \mathrm{d} x$ to leading order, and the defect structure is essentially 
described by the scaling introduced in \$3.4.1. Inspection of $(2.2 a)$ and (3.3) then shows that for any $\epsilon \ll 1, \sigma \ll 1$, the flow in the small-defect region is governed by the expansions

$$
\begin{gathered}
{\left[\frac{U_{\mathscr{S} \eta}-\psi / \delta}{u_{t}}, \frac{-\left\langle u^{\prime} v^{\prime}\right\rangle}{\alpha u_{t}^{2}}\right] \sim[F, \Sigma](x, \eta ; k)+O(\epsilon, \alpha \epsilon), \quad \frac{\delta}{\sigma} \sim \Delta(x ; k)+O(\epsilon),} \\
0<x<x_{\mathscr{D}}, \quad \eta=y / \delta, \quad 0<\eta \leqslant 1 .
\end{gathered}
$$

Here higher-order contributions of $O(\epsilon)$ are triggered by the nonlinearities of the inertia terms in $(2.2 a)$, which, however, appear to be inessential in view of the subsequent investigation.

An investigation of the boundary layer approximation of $(2.2 a)$ involving the firstorder quantities $F, \Sigma, \Delta$ reveals that these can only be matched with the flow quantities in the aforementioned region of rapid transition if $\Sigma$ assumes a finite limit at the base of the defect layer (Scheichl et al. 2008; Scheichl \& Kluwick 2008b, 2009; Kluwick \& Scheichl 2009). This is accounted for by choosing $u_{t}$ such that $\Sigma \rightarrow 1$ as $\eta \rightarrow 0$. Also, as a start, it proves sufficient to only discard the (meaningless) case $\alpha \gg 1$, so that $U_{\mathscr{S}}-u$ predominantly accounts for the velocity defect characteristic of the turbulent boundary layer rather than for absorbing the effect of the surface curvature on the potential flow, as seen from (3.3) and the truncated terms in $(3.13 a)$. Furthermore, the inertia terms are seen to be negligibly small in the viscous wall layer. This allows a direct match of the latter with the defect layer, which is crucial for the particular two-layer structure of the boundary layer. By adopting the basic idea behind the classical theory of (fully developed) wall-bounded turbulent flow, we have in consequence that $u_{t}$ also serves as a suitable reference velocity for both $u$ and the Reynolds stresses in the viscous sublayer. Accordingly, $I_{t}$ measures the magnitude of the Reynolds stresses in both tiers.

As a result of these considerations, the scalings

$$
\left[\frac{u}{u_{t}}, \frac{-\left\langle u^{\prime} v^{\prime}\right\rangle}{\alpha u_{t}^{2}}\right]=\left[u^{+}, \tau_{t}^{+}\right]\left(x, y^{+} ; k, R e\right), \quad y^{+}=\frac{y}{\delta_{v}}, \quad \delta_{v}=\frac{1}{\alpha u_{t} \operatorname{Re}} \sim \frac{1}{\sigma \operatorname{Re} U_{\mathscr{S}}}
$$

are appropriate in the comparatively thin wall layer; note (3.12) and (3.13a). The expression for the wall layer thickness $\delta_{v}$ in (3.14) is underpinned by the form of momentum equation $(2.2 a)$ for $y^{+}=O(1)$ when integrated with respect to $y$ subject to $(2.3)$,

$$
\begin{gathered}
\tau^{+} \sim 1+p^{+} y^{+}+i^{+}\left\{\int_{0}^{y^{+}} \frac{\partial_{x}\left[u_{t} u^{+}(x, t ; k, R e)^{2}\right]}{u_{t}} \mathrm{~d} t-u^{+} \int_{0}^{y^{+}} \partial_{x} u^{+}(x, t ; k, R e) \mathrm{d} t\right\} \\
+O\left(\delta_{v}\right), \quad \tau^{+}=\tau_{t}^{+}+\partial_{y^{+}} u^{+}, \quad i^{+}=\delta_{v} / \alpha, \quad p^{+}=\left(i^{+} / u_{t}^{2}\right) \mathrm{d} p \mathscr{S}(x ; k) / \mathrm{d} x .
\end{gathered}
$$

Here, the dominant contribution to the remainder term is due to the Reynolds normal stress. Most importantly, it is indicated by (3.15) that one conveniently identifies the wall shear stress, given by $\tau_{w}=R e^{-1} \partial u / \partial y$ for $y=0$, with $\alpha u_{t}^{2}$; that is,

$$
\tau_{w} / U_{\mathscr{S}}^{2}=\alpha \gamma^{2} .
$$

To leading order, (3.15) reduces to the expected balance of the sum of the viscous and the Reynolds shear stresses, with the wall shear stress as the pressure gradient contributes to higher-order effects. This situation is enforced by the match with the fully turbulent small-defect flow in the bulk of the boundary layer. Then, both the 
quantities $p^{+}$and $i^{+}$provide asymptotically small gauge functions that control the effects of the pressure gradient and inertia, respectively, in the resulting (formal) expansions,

$$
\begin{aligned}
p^{+} \sim & -\pi^{+} U_{\mathscr{S}}^{-2} \mathrm{~d} U_{\mathscr{S}} / \mathrm{d} x+\cdots, \quad \pi^{+}=\left(\epsilon \sigma^{2} R e\right)^{-1} \ll 1, \\
{\left[u^{+}, \tau^{+}\right] \sim } & {\left[u_{0}^{+}\left(y^{+}\right), 1\right]+p^{+}\left[u_{p}^{+}\left(y^{+}\right), y^{+}\right] } \\
& +i^{+}\left\{\left[u_{i}^{+}, \tau_{i}^{+}\right]\left(x, y^{+} ; k\right)+\cdots+\pi^{+}\left[u_{i p}^{+}, \tau_{i p}^{+}\right]\left(x, y^{+} ; k\right)+\cdots\right\} .
\end{aligned}
$$

For the derivation of $(3.17 a)$, we refer to (3.15), (3.12) and (3.4). In (3.17b), the common key assumption made is that the wall layer flow is in equilibrium: the wall functions $u_{0}^{+}\left(y^{+}\right)$and $u_{p}^{+}\left(y^{+}\right)$are taken as 'universal', i.e. they do not exhibit any dependence on streamwise variations of the flow.

Strictly speaking, $u_{0}^{+}\left(y^{+}\right)$and $u_{p}^{+}\left(y^{+}\right)$have to be determined experimentally or via DNS. The only information available in an asymptotic flow description using turbulence-closure-free RANS is the broadly believed behaviour

$$
-\left\langle u^{\prime} v^{\prime}\right\rangle=O\left(y^{+3}\right), \quad y^{+} \ll 1,
$$

which is supported by evaluating the continuity equation for the fluctuating motion for $y=0$ in combination with (2.3) (cf. Monin \& Yaglom 1971, pp. 270-272), and the conditions of matching with the small-defect flow on top of the viscous sublayer. These basic considerations yield the limiting representations

$$
\begin{aligned}
y^{+} \rightarrow 0: & {\left[u_{0}^{+}, u_{p}^{+}\right] \sim\left[y^{+}, y^{+2} / 2\right]+O\left(y^{+4}\right), } \\
y^{+} \rightarrow \infty: & u_{0}^{+} \sim \kappa^{-1} \ln y^{+}+C^{+}, \quad \kappa \doteq 0.384, \quad C^{+} \doteq 4.173 .
\end{aligned}
$$

The relationship (3.19b) represents the celebrated logarithmic law of the wall. The (currently accepted) empirical values for the von Kármán constant $\kappa$ and the intercept $C^{+}$have been reported by Nagib, Chauhan \& Monkewitz (2007). Although found for the canonical zero-pressure-gradient boundary layer, they seem admissible in the present context in view of the asymptotic equilibrium expressed through $(3.17 b)$.

The above considerations complete the description of the flow in the outer tier. Integrated once with respect to $\eta$, the leading-order boundary layer equation then reads

$$
U_{\mathscr{S}}^{-1} \partial_{x}\left(U_{\mathscr{S}} \Delta\right) \eta \partial_{\eta} F-U_{\mathscr{S}}^{-3} \partial_{x}\left(U_{\mathscr{S}}^{3} \Delta F\right)=\Sigma-1 .
$$

Equation (3.20) is subject to the conditions of matching and patching with the flow representations in the wall region and the irrotational external flow, respectively, for

$$
\begin{array}{ll}
\eta \rightarrow 0: & F \rightarrow 0 \quad(\Sigma \rightarrow 1), \quad \partial_{\eta} F \sim-\kappa^{-1} \ln \eta+C(x ; k), \\
\eta=1: & \partial_{\eta} F=\partial_{\eta}^{2} F=\Sigma=0 .
\end{array}
$$

With respect to the further analysis, it is interesting to note that the scaling velocity $u_{t}$ prevents higher-order terms in the expansion of $F$ provided by $(3.13 a)$ from exhibiting a logarithmic singularity similar to that given in $(3.21 a)$. The function $C(x ; k)$ depends on the actual turbulence closure adopted to model $\Sigma$. The condition for $\partial_{\eta}^{2} F$ imposed at the boundary layer edge is due to vanishing vorticity. Also, (3.20) is supplemented with universal initial conditions, as predicted by (3.20) and (3.7a) and revealed from the limiting form for

$$
x \rightarrow 0_{+}: \quad[F, \Sigma](x, \eta ; k) \sim\left[F_{0}, \Sigma_{0}\right](\eta)+O(x), \quad[\Delta / x, C](x ; k) \sim\left[\Delta_{0}, C_{0}\right]+O(x) .
$$


Here the subscript 0 indicates the stagnant-flow solution that describes the terminal stage of the laminar-turbulent transition process as treated in $\$ 3.4 .1$, and in more length and breadth by Scheichl et al. (2008). The values of the quantities $F_{0}, \Sigma_{0}$, and the (positive) constants $\Delta_{0}, C_{0}$ are the solutions of the boundary value problem

$$
\begin{aligned}
& 2 \Delta_{0}\left(\eta F_{0}^{\prime}-2 F_{0}\right)=\Sigma_{0}-1, \\
\eta \rightarrow 0: \quad F_{0} \rightarrow 0, \quad F_{0}^{\prime} & \sim-\kappa^{-1} \ln \eta+C_{0}, \quad \eta=1: \quad F_{0}^{\prime}=F_{0}^{\prime \prime}=\Sigma_{0}=0,
\end{aligned}
$$

representing the limiting forms of (3.20) and (3.21) as $x \rightarrow 0_{+}$; here and in the following, primes on $F$ denote ordinary derivatives with respect to the independent variable.

Finally, matching the small-defect and the wall layer according to (3.19b), (3.21a) and and (3.12) yields

$$
\gamma / \epsilon \sim 1-\epsilon\left[\kappa^{-1} \ln \left(U_{\mathscr{S}} \Delta\right)+C+C^{+}\right]+O\left(\epsilon^{2}\right), \quad \epsilon=\kappa / \ln \left(\sigma^{2} R e\right) \ll 1,
$$

which determines $u_{t}$ (in agreement with the asymptotic errors anticipated in $(3.13 a)$ ), provides a first relationship between $\epsilon, \sigma$ and $R e$, and, in view of (3.16), represents the skin-friction law. It is noted that (3.24) confirms that the pressure gradient affects the wall layer equilibrium only in second order, as anticipated in (3.15) and (3.17).

\subsubsection{Boundary layer scaling: $T$ - versus $(\epsilon, \sigma)$-formalism}

In the first instance, (3.24) asserts that $\delta$ is much larger than $R e^{-1 / 2}$, measuring the thickness of a strictly laminar or a laminar-turbulent boundary layer (a notion introduced in $\S 3$ ) having $T=O(1)$. Let us reconsider the match of $\bar{\Sigma}$ for $x=O\left(T^{-1}\right)$, defined by (3.10), and for $x=O(1)$, where it has the form given by (3.12). Without loss of generality, we assume $\bar{\Sigma} / u_{t}^{2} \sim \Sigma_{0}=O(1)$ in the intermediate regime $T^{-1} \ll x \ll 1$ according to $(3.13 a)$ and $(3.22)$ and thereby readily reveal by using (3.11) the existence of an underdeveloped turbulent boundary layer flow as

$$
\alpha=T R e^{-1 / 2} \ll 1 \text {. }
$$

This relationship, backed by the proposed specific picture of short-scale transition path to turbulence, underlies (1.3b). From (1.2) and (3.24) then follows

$$
\epsilon \sim \epsilon_{T}=\kappa /(2 \ln T), \quad \sigma \sim \alpha \epsilon_{T}, \quad I_{t} \sim \alpha \epsilon_{T}^{2},
$$

which completes the scaling of the boundary layer in terms of $T$ and $R e$, as proposed originally by Neish \& Smith (1992). Hence, for $T \gg 1$, the boundary layer thickness is augmented in magnitude by the factor $\epsilon_{T} T$ whereas that of the emerging wall layer is reduced by the same factor when compared to the thickness of a laminar-turbulent boundary layer mentioned above.

Also, first conclusions that prove useful for the subsequently preferred description of the interactive boundary layer flow in terms of the asymptotic parameters $\epsilon$ and $\sigma$ are drawn from (3.24): when the value of $I_{t}$ is increased for Re kept fixed, then $\sigma$ (i.e. $\delta$ ) increases and $\epsilon$, the wall layer thickness $\delta_{v}$ given by (3.14) and in turn the von Kármán number,

$$
\delta^{+}=\delta_{v} / \delta \sim 1 /\left(\sigma^{2} \operatorname{Re}_{\mathscr{S}} \Delta\right),
$$

decrease as $\mathrm{d}[\sigma, \epsilon] / \mathrm{d} I_{t} \sim[1 / \epsilon,-2 \epsilon /(\kappa \sigma)]$. A possibly appealing aspect of these findings is noted, namely that they allow 'stimulation' of the variations of $I_{t}$ and $\epsilon$ by passing through all possible dependences of $\sigma$ on $R e$ as $\sigma$ is increased. If $\sigma^{2} R e$ is sufficiently large to vary predominantly algebraically with $R e$, so that $\delta^{+}$varies accordingly with some negative power of $R e$, see (3.14), we already arrive at the 


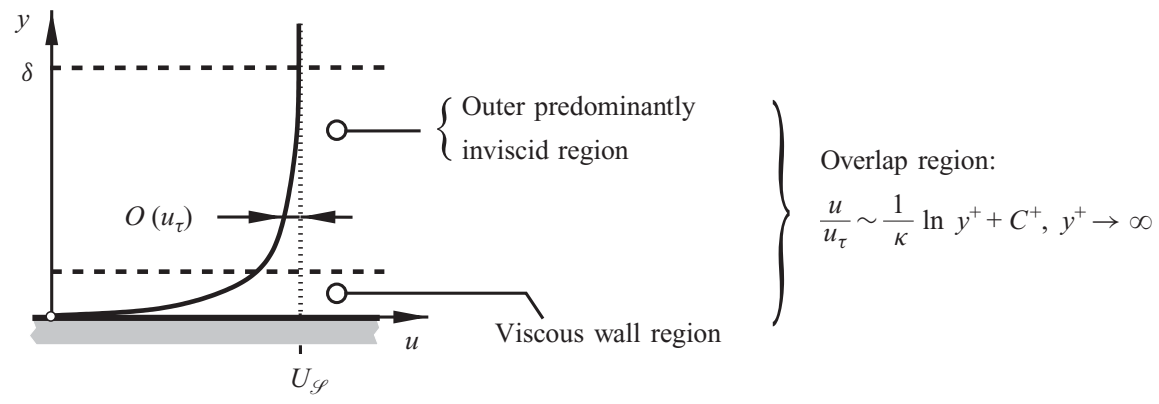

FIGURE 3. Asymptotic substructures of incident boundary layer and overlap behaviour (3.19b) (not to scale, for labelling see text).

traditional asymptotic scaling of the velocity defect as $\epsilon=O(1 / \ln R e)$. Then, we are concerned with the precise definition of slightly underdeveloped turbulent boundary layer flow, so that (1.3) supplemented with (3.24) now is expressed in the more accurate form

$$
\sigma=\chi(R e) R e^{\mu-1 / 2}, \quad \epsilon \sim \kappa /(2 \mu \ln R e), \quad \text { with } \quad \ln \chi=o(\ln \operatorname{Re}), \quad 0<\mu<1 / 2 .
$$

Here the slowly varying gauge function $\chi(R e)$ and the exact value of the constant $\mu$ are determined by the inner mechanism of viscous-inviscid interaction and eventually provide an accurate estimate of the value of $I_{t}$ in the regime (1.1) as it fixes the dependences of $\epsilon$ and $\alpha$ on $R e$.

We revert to this issue in $\S 5.1$ in more depth. Here we only note that the relationships (3.27) conform to the internal scaling of the associated triple-deck structure, as anticipated in $\S 1$ : any further increase of $\sigma$ (or, equivalently, of $I_{t}$ ) that is accompanied by a weaker, i.e. not basically algebraic, rate of decay of $\sigma$ for increasing values of $R e$ is precluded, as it implies super-algebraic growth of $R e$ in the dependence on $1 / \sigma$ and, in turn, a correspondingly strong unacceptable decay of $\delta^{+}$ in the dependence on $\sigma$, according to (3.26). Eventually, the hypothetical upper and lower limits of $\sigma$ (i.e. of $I_{t}$ ) and $\epsilon$, respectively, which refer to the classical (i.e. fully developed) turbulent boundary layer, are attained when $\mu$ assumes its least upper bound given by $1 / 2$ and for $\sigma=\chi$ chosen proportional to $\epsilon$, i.e. for $\alpha=O(1)$. This entails $\epsilon \sim \kappa / \ln R e$, a situation exactly unmasking the scaling of the classical (i.e. fully developed) turbulent boundary layer. Then $u_{t}$ serves as the single reference quantity for scaling both the velocity defect and the Reynolds stresses, see $(3.13 a)$. In that case, $\delta^{+}$ varies with $\exp (-\kappa / \epsilon) / \epsilon^{2}$ or, equivalently, with $(\ln R e)^{2} / R e$, in agreement with (3.14), which expresses the well-established property that the wall layer is transcendentally thin when compared with the outer defect layer.

Figure 3 depicts the resulting two-tiered structure of the attached turbulent boundary layer. Next, we study its asymptotic subdivision when the boundary layer flow approaches the point $\mathscr{D}$ of inviscid flow detachment, i.e. when exposed to a free-stream speed $U_{\mathscr{S}}$ that locally has the form $(3.7 b)$.

\section{Non-interactive boundary layer near separation}

The flow behaviour in the outer main (small-defect) part of the boundary layer as $x \rightarrow x_{\mathscr{D}}$ is important for the local separation process. Below, particular emphasis is 
placed on a further sublayer that emerges as the main part splits, before we address the viscous wall layer.

\subsection{Small-defect layer}

It has been pointed out by Scheichl \& Kluwick $(2008 a, b)$ that the velocity defect, scaled Reynolds stresses and the boundary layer thickness take on finite limits with

$$
\begin{aligned}
\{[F, \Sigma], \Delta\} \sim & \left\{\left[F_{\mathscr{D}}, \Sigma_{\mathscr{D}}\right](\eta ; k), \Delta_{\mathscr{D}}(k)\right\}+(-s)^{1 / 2}\left\{\left[F_{1 / 2}, \Sigma_{1 / 2}\right](\eta ; k), \Delta_{1 / 2}(k)\right\} \\
& +(-s)\left\{\left[F_{1}, \Sigma_{1}\right](\eta ; k), \Delta_{1}(k)\right\}+O\left[(-s)^{(3 / 2)}\right] \text { as } s \rightarrow 0_{-},
\end{aligned}
$$

where $s$ is defined by (3.2) and the quantities with subscripts are $O(1)$, from (3.20) and (3.21). The quantities with subscript $1 / 2$ are unaffected explicitly by the Reynolds shear stress. For the equations determining the quantities with subscript $1 / 2$ and 1 , one infers from the requirement $F_{1 / 2}^{\prime \prime}(1 ; k)=F_{1}^{\prime \prime}(1 ; k)=0$ (by disregarding the rather unlikely case $\left.F_{\mathscr{D}}^{\prime \prime \prime}(1 ; k)=0\right)$, see $(3.21 b)$, the solvability conditions (cf. Scheichl \& Kluwick 2008a,b)

$$
\Delta_{1 / 2}=-2 k \Delta_{\mathscr{D}}, \quad \Delta_{1}=\Delta_{\mathscr{D}}\left(2 k^{2} / 3-\beta\right), \quad \beta=\Sigma_{\mathscr{D}}^{\prime \prime}(1 ; k) /\left[F_{\mathscr{D}}^{\prime \prime \prime}(1 ; k) \Delta_{\mathscr{D}}\right](>0) .
$$

Consequently, one obtains

$$
F_{1 / 2}=-4 k F_{\mathscr{D}}, \quad F_{1}=-\beta \eta F_{\mathscr{D}}^{\prime}+\left(60 k^{2} / 3+\beta\right) F_{\mathscr{D}}+\Sigma_{\mathscr{D}} / \Delta_{\mathscr{D}} .
$$

The results (4.2) and (4.3) involve terms already found for the contributions of $O\left[(-s)^{1 / 2}\right]$. Also, they give values of $\Sigma_{1 / 2}, \Sigma_{1}$ according to the chosen Reynolds stress model and the corresponding linearisation of the shear rate $F^{\prime \prime}$ about $F_{\mathscr{D}}^{\prime \prime}$. Higherorder terms in (4.1) can be determined by proceeding in this manner. Eventually, we find from (3.13a), together with $(3.7 b)$ and $(1.3 b)$, that the velocity defect locally has the form

$$
\left(U_{\mathscr{S}}-u\right) / u_{0 \mathscr{D}} \sim \epsilon F_{\mathscr{D}}^{\prime}\left[1-2 k(-s)^{1 / 2}+O(-s)\right]+O\left(\epsilon^{2}\right) .
$$

Since $F_{\mathscr{D}}$ is subject to $(3.21 a)$ for $x=x_{\mathscr{D}}$, each member of the sequence $F_{1 / 2}, F_{1}, \ldots$, exhibits a similar logarithmic singularity (as indicated by (4.3)), which violates the original boundary conditions $(3.21 a)$ for $F$. This inconsistency shows that the initialboundary-value problem posed by (3.20)-(3.23) is singularly perturbed as $s \rightarrow 0_{-}$. Thus, a small so-called adjustment region arises where $\eta=O(-s)$, so that the balance of linearised convection with the Reynolds stress gradient expressed by (3.20) is retained to leading order. We accordingly define

$$
[F /(-s), \Sigma]=[\hat{F}, \hat{\Sigma}](s, \hat{\eta} ; k), \quad \hat{\eta}=\eta /(-s)=O(1), \quad s \rightarrow 0_{-},
$$

and end up with the appropriate expansions

$$
\begin{array}{rlrl}
F_{\mathscr{D}} \sim & \hat{F}_{\mathscr{D}}(\eta ; k)=\left[C_{\mathscr{D}}+\kappa^{-1}(1-\ln \eta)\right] \eta, \quad C_{\mathscr{D}}=C\left(x_{\mathscr{D}} ; k\right), & \eta \rightarrow 0, \\
{[\hat{F}, \hat{\Sigma}] \sim\left[-\kappa^{-1} \hat{\eta} \ln (-s)+\hat{F}_{\mathscr{D}}(\hat{\eta} ; k), 1\right]} & & \\
& +(-s)^{1 / 2}\left[4 k \kappa^{-1} \hat{\eta} \ln (-s)+\hat{F}_{1}(\hat{\eta} ; k), \hat{\Sigma}_{1}(\hat{\eta} ; k)\right]+\cdots, \quad s \rightarrow 0_{-} .
\end{array}
$$

The quantities $\hat{F}_{1}, \hat{\Sigma}_{1}$ describe the dominant deviations from the behaviour determined by $(3.21 a)$ that governs $\hat{F}, \hat{\Sigma}$, and they satisfy the correspondingly reduced form of $(3.20)$,

$$
-\hat{\eta} \hat{F}_{1}^{\prime}+(3 / 2) \hat{F}_{1}=\hat{\Sigma}_{1}+2 k \hat{\eta}\left[\kappa^{-1}(\ln \hat{\eta}-3)-C_{\mathscr{D}}\right] .
$$

The matching with the quantities in the main region where $\eta$ is $O(1)$ and the conditions to resolve the mismatch with the wall layer flow give

$$
\hat{\eta} \rightarrow \infty: \quad \hat{F}_{1} \sim \hat{a}(k)\left[(2 / 3) \hat{\eta}^{3 / 2}+\cdots\right]-4 k \hat{F}_{\mathscr{D}}(\hat{\eta} ; k)+\cdots,
$$




$$
\hat{\eta} \rightarrow 0: \quad \hat{F}_{1}=\hat{\Sigma}_{1}=0, \quad \hat{F}_{1 \hat{\eta}}=O(1) .
$$

The terms proportional to the unknown function $\hat{a}(k)$ are due to the homogeneous part of $(4.7 a)$. The second contribution to $(4.7 b)$ arises from the particular solution of $(4.7 a)$ and provides consistency with $(4.6 a)$. The variation of the streamwise velocity component with the square root of wall distance on top of the logarithmic region here is similar to that in the immediate vicinity of comparatively 'mild', i.e. marginal, turbulent separation (Scheichl \& Kluwick 2007a,b), even though the local flow description in these studies closely resembles that of a turbulent boundary layer in quasi-equilibrium, showing global internal separation, i.e. globally reversed flow (cf. Schlichting \& Gersten 2003).

It is widely believed that on top of the viscous wall layer, the mixing-length model of Prandtl holds,

$$
-\left\langle u^{\prime} v^{\prime}\right\rangle \sim(\kappa y)^{2} \partial_{y} u\left|\partial_{y} u\right|
$$

where it predicts the logarithmic velocity distribution as a result of the approximately constant shear stress (cf. Schlichting \& Gersten 2003). If (4.8) is chosen as a specific shear stress closure for the adjustment region, we then arrive at $\hat{\Sigma}=\left(\kappa \hat{\eta} \partial_{\hat{\eta}}^{2} \hat{F}\right)^{2}$ and, by linearisation in view of (4.6),

$$
\hat{\Sigma}_{1}=2 \kappa \hat{\eta} \partial_{\hat{\eta}}^{2} \hat{F}_{1}
$$

In turn, one recognises that only $(4.7 c)$ represents a true boundary condition for $(4.7 a)$, and $(4.7 b)$ is satisfied identically. In fact, for any value of $\hat{a}$ in $(4.7 b)$, the two homogeneous solutions of (4.7a) and (4.9) are represented by the dominant algebraic behaviour proportional to $\hat{a}$, so that the dots in square brackets in $(4.7 b)$ stand for $-\hat{\eta}^{1 / 2}+O\left(\hat{\eta}^{-1 / 2}\right)$ and terms that vary basically with $\exp [-\hat{\eta} /(2 \kappa)]$. In consequence, $(4.7 b)$ is supplemented with $\hat{\Sigma} \sim \hat{a} \hat{\eta}^{1 / 2}+8 k+O\left(\hat{\eta}^{-1 / 2}\right)$, as $\hat{\eta} \rightarrow \infty$, which matches $\Sigma_{\mathscr{D}}$ and $\Sigma_{1 / 2}$ in (4.1), and we obtain a two-term expansion at the base of the defect region close to separation,

$$
F_{\mathscr{D}} \sim \hat{F}_{\mathscr{D}}+(2 / 3) \hat{a}(k) \eta^{3 / 2}+\cdots, \quad \Sigma_{\mathscr{D}} \sim\left(\kappa \eta F_{\mathscr{D}}^{\prime \prime}\right)^{2} \sim 1+\hat{a}(k) \kappa \eta^{1 / 2}+\cdots, \quad \eta \rightarrow 0 .
$$

Therefore, $\hat{a}(k)$ is determined by the overall solution of the boundary layer problem upstream of $x=x_{\mathscr{D}}$. Moreover, we deduce from (4.9) the near-wall behaviour

$$
\hat{F}_{1} \sim \hat{b} \hat{\eta}-k /\left(2 \kappa^{2}\right) \hat{\eta}^{2} \ln \hat{\eta}+\left[\hat{b} /(8 \kappa)+k\left(9+2 \kappa C_{\mathscr{D}}\right) /\left(4 \kappa^{2}\right)\right] \hat{\eta}^{2}+O\left(\hat{\eta}^{3} \ln \hat{\eta}\right), \quad \hat{\eta} \rightarrow 0,
$$

where $\hat{b}=\hat{F}_{1}(0 ; k)$ depends linearly on $\hat{a}$. The absence of a logarithmic behaviour in $\hat{F}_{1 \hat{\eta}}$ for $\hat{\eta} \rightarrow 0$ a posteriori justifies the introduction of the sublayer. Furthermore, from (4.6) the velocity defect at the base of the small-defect region is increased by an amount of $O\left[(-s)^{1 / 2} \ln (-s)\right]$ as

$$
C \sim C_{\mathscr{D}}+(-s)^{1 / 2}\left[4 k \kappa^{-1} \ln (-s)+\hat{b}\right]+O[(-s) \ln (-s)], \quad s \rightarrow 0_{-} .
$$

Finally, problem (4.7) supplemented with (4.9) can be solved in closed form for a given $\hat{a}(k)$. To this end, we conveniently decompose $\hat{F}_{1}(\hat{\eta} ; k)=\hat{F}_{1 h}+\hat{F}_{1 p}$, where

$$
\begin{aligned}
\hat{F}_{1 h}(\hat{\eta} ; k)= & \mathrm{e}^{-\check{\eta}} \check{\eta}\left\{\hat{a}(k) \sqrt{\kappa^{3} \pi / 2}\left[(4 \check{\eta}+3) \mathrm{I}_{0}(\check{\eta})+(4 \check{\eta}+1) \mathrm{I}_{1}(\check{\eta})\right]\right. \\
& +(4 k / 3)\left[(4 \check{\eta}+3) \mathrm{K}_{0}(\check{\eta})-(4 \check{\eta}+1) \mathrm{K}_{1}(\check{\eta})\right\}, \quad \check{\eta}=\hat{\eta} /(4 \kappa),
\end{aligned}
$$

represents a homogeneous solution of (4.7a) expressed in terms of the modified Bessel functions $\mathrm{I}_{0}, \mathrm{I}_{1}$ and $\mathrm{K}_{0}, \mathrm{~K}_{1}$ of the first and second kinds, respectively, and

$$
\hat{F}_{1 p}(\hat{\eta} ; k)=16 k / 3-4 k \hat{F}_{\mathscr{D}}(\hat{\eta} ; k)
$$

a particular one. 


\subsection{Numerical results}

We now demonstrate that the results of $\S 4.1$ are in fair agreement with numerical solutions of the boundary layer problem posed by (3.20)-(3.23). Here we consider the canonical H-K flow around a circular cylinder of radius $\tilde{L}$, equal to 1 in nondimensional form. In particular, we focus on the behaviour of the key quantities $U_{\mathscr{S}}(x ; k)$ and $\Delta(x ; k)$. The specific form of $F_{\mathscr{D}}(Y ; k)$ is part of the solutions, parametrised by the potential flow parameter $k$.

Solving this problem represents the only stage in the present analysis where the Reynolds shear stress has to be modelled explicitly in the entire bulk of the boundary layer, but without affecting any of the results of the analysis qualitatively. We close these equations by adopting the traditional formalism based on the mixing length $\ell$, which is defined for $0 \leqslant y \leqslant \delta$ by setting $-\left\langle u^{\prime} v^{\prime}\right\rangle=\ell^{2} \partial_{y} u\left|\partial_{y} u\right|$, with $\ell=0$ for $y=0$, $\ell>0$ for $y>0$, and $\ell$ being finite for $y=\delta$. This approach is then consistent with the conditions $(3.21 b)$ of smooth patching with the external flow, and the matching conditions (3.21a) are accounted for when we assume that (4.8) holds in the overlap of the defect and the viscous wall layer, viz. $\ell \sim \kappa y$. The algebraic closure by Michel, Quémard \& Durant (1969), utilised quite frequently in the literature on turbulent boundary layers, provides a most simple but sufficiently complex example for such an asymptotically correct modelling. Specifically, here we employ a slight modification of the model in the form

$$
\Sigma=\left[(\ell / \delta) \partial_{\eta}^{2} F\right]^{2}, \quad \ell / \delta=c_{\ell} I_{K}^{1 / 2} \tanh \left(\eta \kappa / c_{\ell}\right), \quad I_{K}=1 /\left(1+5.5 \eta^{6}\right), \quad c_{\ell}=0.085,
$$

where the inclusion of the intermittency factor $I_{K}(\eta)$ proposed by Klebanoff (1955) is expected to cope with the usually observed overestimate of $\Delta$ and $\Sigma$ near the boundary layer edge (when compared with results obtained with eddy-viscosity-based models). We use the value of $\kappa$ noted in (3.19b).

In order to obtain a highly accurate solution of (3.20)-(3.23), supplemented with (4.14), we adopted an advanced numerical scheme with a Keller-Box discretisation, combined with the method of lines with respect to the streamwise direction, and an automatic adaptive remeshing strategy. Downstream integration is performed by using a higher-order backward-differentiation-formula technique or alternatively a Theta method, where the tolerance $e_{m}$ of the (locally estimated) absolute error is given by $m^{1 / 2}$, with $m$ denoting the relative machine precision ( $m \approx 10^{-16}$ in our case). It is initiated by solving (3.23) with the local absolute error bounded by $e_{m}$. This is accompanied by the automated generation of a non-uniform initial mesh for the variable $\eta$, having typically 550 nodes optimally distributed in the interval $\eta_{0} \leqslant \eta \leqslant 1$, where $\eta_{0}=10^{-5}$ (lower values of $\eta_{0}$ result in severe numerical difficulties due to the logarithmic singularity in (3.21)). Therefore, the associated methodical error matches the numerical error expected to be encountered in the calculation of the H-K flow, as explained in $\S$ B.3 (Appendix B): both $U_{\mathscr{S}}(x ; k)$ and $\mathrm{d} U_{\mathscr{S}} / \mathrm{d} x$ are imposed by evaluation of two cubic B-splines that interpolate $U_{\mathscr{S}}$ and $x$ between values of these quantities computed for uniformly spaced values of the auxiliary variable $\varrho$. Consequently, the spacing between consecutive $x$-values is properly condensed near the B-V singularity, according to (B 6). A typical number of 450 spline knots for $0 \leqslant x \leqslant x_{\mathscr{D}}(-\pi / 2 \leqslant \varrho \leqslant 0)$ renders the additional error due to the spline interpolation sufficiently small. We note that the boundary layer problem is singular not only for $x=x_{\mathscr{D}}$ but also for $x=0$, as is the convective operator in (3.20) due to the disappearance of both $U_{\mathscr{S}}$ and $\Delta$; see (3.7a) and (3.22). Accordingly, the interval of downstream integration is given by $e_{m} \leqslant x \leqslant x^{*}$, 
$x_{\mathscr{D}}-x^{*}=\Delta x>0$, with $\Delta x$ having the magnitude of $e_{m}$, which proves satisfactorily accurate. It should be noted that the value $x=x^{*}$ of termination of the solution is largely affected by the internal stopping criteria implemented in the integrator used (which apparently impedes a higher resolution of the results close to $x=x_{\mathscr{D}}$ ).

We discuss the results for $k=0.45$ as an example, which yields $x_{\mathscr{D}}(0.45) \doteq 1.9849 \doteq 113^{\circ} 43^{\prime} 28^{\prime \prime}$ and represents a physically reliable choice in view of the semi-empirical knowledge of bluff-body separation in the regime (1.1) (cf. Zdravkovich 1997). It is found that $C_{0} \doteq 1.3212, \Delta_{0} \doteq 0.07166$, and (by extrapolation to $\left.x=x_{\mathscr{D}}\right) C_{\mathscr{D}} \doteq 8.1760, \Delta_{\mathscr{D}} \doteq 0.4464$.

Figure $4(a, b)$ shows the solutions for $\partial_{\eta} F$ and $\Sigma$ : the defect profiles become more and more pronounced (i.e. they deviate from the logarithmic law in the overlap as $C$ increases, see $(3.21 a)$ ) the more the boundary layer approaches separation. Simultaneously, the position where the Reynolds shear stress assumes its maximum moves from the overlap region outwards into the defect layer. Such shear stress profiles are typically observed in any type of separating turbulent boundary layer. Also, the smooth patching with the external flow as $\left[F^{\prime}, \Sigma\right]=O\left[(1-\eta)^{2}\right]$ is clearly visible, and we note that $\Sigma-1=O(\eta \ln \eta)$ as $\eta \rightarrow 0$ (deduced from (3.20) and (3.21a)). Note that here $x_{\mathscr{D}}$ is identified with $x^{*}$. In figure $4(c)$, the values of the key quantities $U_{\mathscr{S}}$ and $\Delta$, including their asymptotes (see (3.22), (4.1) and (4.2)) and the coefficient $c_{p \mathscr{S}}$ of the surface pressure, $c_{p \mathscr{S}}=1-U_{\mathscr{S}}^{2}$, are plotted.

Finally, the $\log -\log$ plots in figure 5 display a comparison of the numerical with the asymptotic results holding at the onset of separation. This exploits the twoterm expansion (4.1) with $\left[g_{1}, g_{2}\right]=\left[2 k(-s)^{1 / 2},(-s)\right]$ and $\left[\Delta_{1}, \Delta_{2}\right]=\left[\Delta g_{1} /\left(1-g_{1}\right)\right.$, $\left.\Delta_{\mathscr{D}}\left(1-g_{1}\right)-\Delta\right]$ serving as gauge functions and reference quantities (here the upright Delta symbols indicate 'difference') respectively. The behaviours $\Delta_{1} \sim \Delta_{\mathscr{D}} g_{1}+O\left(g_{1}^{2}\right)$, $\Delta_{2} \sim-\Delta_{1} g_{2}+O\left(g_{2}^{3 / 2}\right)$ are easily verified. Here the asymptote of the first relationship is reproduced excellently, whereas that of the second suffers a more distinct deviation from the numerical data due to the relatively larger effect of higher-order terms. The closure-dependent (negative) value of the coefficient $\Delta_{1}$ is confirmed by evaluation of (4.2).

\subsection{Wall layer}

To complete the description of the non-interactive flow, the behaviour of the wall layer flow approaching $\mathscr{D}$ has to be investigated next. One infers from (3.15), (3.17) and (3.24) that

$$
\begin{aligned}
\tau_{i}^{+} & =\frac{U_{\mathscr{S}}^{\prime}}{U_{\mathscr{S}}} \int_{0}^{y^{+}} u_{0}^{+2}(t) \mathrm{d} t, \quad U_{\mathscr{S}}^{\prime}=\frac{\mathrm{d} U_{\mathscr{S}}(x ; k)}{\mathrm{d} x} \\
\tau_{i p}^{+} & =\frac{\mathrm{d}}{\mathrm{d} x}\left[\frac{U_{\mathscr{S}}^{\prime}}{U_{\mathscr{S}}^{2}}\right] u_{0}^{+} \int_{0}^{y^{+}} u_{p}^{+}(t) \mathrm{d} t-\frac{1}{U_{\mathscr{S}}} \frac{\mathrm{d}}{\mathrm{d} x}\left[\frac{U_{\mathscr{S}}^{\prime}}{U_{\mathscr{S}}}\right] \int_{0}^{y^{+}} u_{0}^{+}(t) u_{p}^{+}(t) \mathrm{d} t .
\end{aligned}
$$

Expanding $p^{+}, \tau_{i}^{+}, \tau_{i p}^{+}$for $s \rightarrow 0_{-}$according to $(3.7 b)$ gives rise to corresponding subexpansions in $(3.17 b)$.

\subsubsection{First breakdown}

The wall layer has a thickness $O[1 /(\sigma R e)]$, according to (3.14), and is thus expected to merge with the adjustment region discussed in $\S 4.1$, when $\delta_{v} \ll-s=O\left[1 /\left(\sigma^{2} R e\right)\right]$ by (3.14). Hence, the flow in this region of the first breakdown of the original wall layer structure is again of boundary layer type. It is characterised by perturbations of the original leading-order stress balance that introduce corresponding variations 

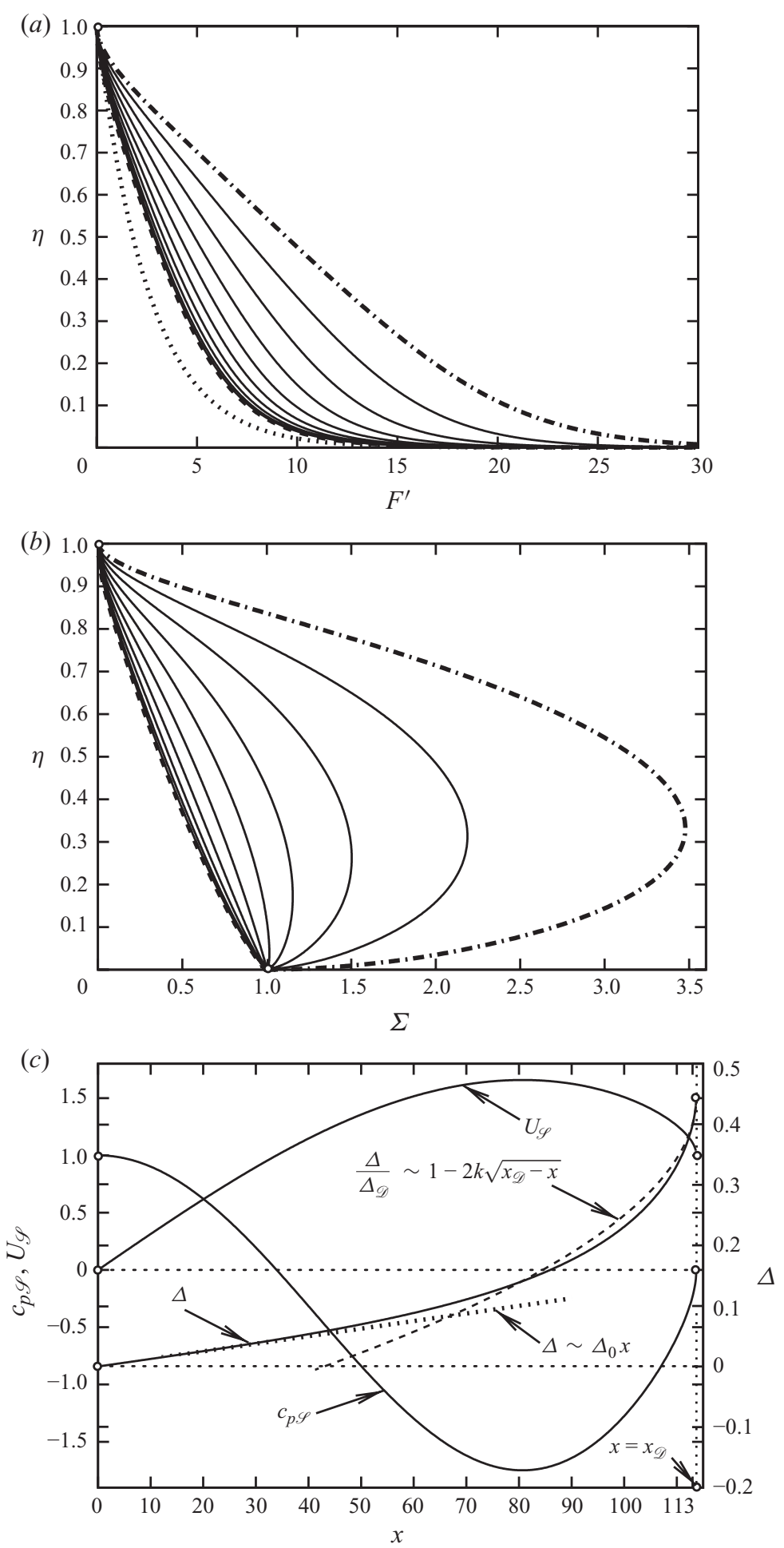

FIGURE 4. Solution of (3.20)-(3.23) for $k=0.45$ (for labelling see text). ( $a, b)$ $-\cdots, \quad x \doteq 0 \quad$ (stagnation); $-, \quad x=x_{\mathscr{D}}\left[1-(i / 10-1)^{2}\right], \quad i=1,2, \ldots, 9$ (curves from left to right refer to increasing values of $i$ ); $\cdots . \cdot, x=x^{*} \doteq x_{\mathscr{D}}$ (incipient separation); $\cdots .$. , asymptote $F^{\prime} \sim-\kappa^{-1} \ln \eta ;(c)$ key quantities. 



FiguRE 5. Comparison of numerical and asymptotic errors of $\Delta$ near $x=x_{\mathscr{D}}$ (for labelling see text). Symbols and lines: $\triangle$, data points; - , asymptotes; $(a)$ first order, -....- (median line); (b) second order, - - - $\left(-\Delta_{1} \pm 0.1\right) g_{2}$.

of the wall shear stress. At present, the detailed structure of this collapse is not completely understood but does not affect the essential characteristics of the second breakdown further downstream.

\subsubsection{Second breakdown}

To the order of accuracy considered here, the wall layer analysis which neglects the interplay with the small-defect flow, resulting in the first breakdown, agrees with that performed by Scheichl \& Kluwick (2008b) in the different context of (3.10) for $T \gg 1$. Thus, it is not repeated here but it suffices to recast the central findings in terms of the perturbation parameters $\epsilon, \sigma$ by using the relationships (3.12) and (3.25) as follows.

The original asymptotic hierarchy reflecting the shear stress balance and the effects of pressure gradient and inertia on the oncoming wall layer flow breaks down when the last two terms become of the same order of magnitude. As seen from $(3.17 b)$ and (4.15), this structural change of the near-wall flow occurs when $i^{+} \tau_{i p}^{+}$, which grows like $(-s)^{-3 / 2}$, is $O\left[(-s)^{-1 / 2}\right]$, i.e. when $\bar{s}=s / \bar{\delta}=O(1)$, with

$$
i^{+} \sim \bar{\delta}=\epsilon /\left(u_{0 \mathscr{D}} \sigma^{2} R e\right) \gg \delta_{\nu}, \quad \bar{s} \rightarrow 0_{-},
$$

here we refer to (3.15), (3.14) and (1.3b). That is, this second breakdown of the wall layer takes place in a region of boundary layer type on a streamwise scale $O(\epsilon)$ smaller than that characterises the first breakdown.

Let the new perturbation parameter

$$
\bar{\pi}^{+}=\pi^{+} k /\left(\bar{\delta}^{1 / 2} u_{0 \mathscr{D}}\right)=k /\left(u_{0 \mathscr{D}}^{1 / 2} \epsilon^{3 / 2} \sigma R e^{1 / 2}\right) \ll 1,
$$

as suggested by (3.15) and (3.17a), account for the pressure rise as $p^{+} \sim \bar{\pi}^{+}(-\bar{s})^{-1 / 2}+\cdots$ for $0<-\bar{s}=O(1)$. Substitution of the appropriate expansion $\left[u^{+}, \tau_{t}^{+}\right] \sim\left[u_{0}^{+}\left(y^{+}\right), 1\right]+\bar{\pi}^{+}\left[\bar{u}^{+}, \bar{\tau}^{+}\right]\left(\bar{s}, y^{+}\right)+O\left(\epsilon^{2} \bar{\pi}^{+}\right)$into (3.15) finally yields to second order the linear boundary layer problem

$$
\begin{gathered}
u_{0}^{+} \frac{\partial \bar{u}^{+}}{\partial \bar{s}}-\frac{\mathrm{d} u_{0}^{+}}{\mathrm{d} y^{+}} \int_{0}^{y^{+}} \frac{\partial \bar{u}^{+}}{\partial \bar{s}}(\bar{s}, t) \mathrm{d} t=-\frac{1}{\sqrt{-\bar{s}}}+\frac{\partial \bar{\tau}^{+}}{\partial y^{+}}, \quad \bar{\tau}^{+}=\bar{\tau}_{t}^{+}+\frac{\partial \bar{u}^{+}}{\partial y^{+}}, \\
y^{+}=0: \quad \bar{u}^{+}=\bar{\tau}_{t}^{+}=0, \quad y^{+} \rightarrow \infty: \bar{u}^{+} \sim \frac{2 \kappa \sqrt{-\bar{s}}}{\ln y^{+}}, \quad \frac{\partial \bar{\tau}_{t}^{+}}{\partial y^{+}} \rightarrow 0 \\
y^{+}=O(1), \quad \bar{s} \rightarrow-\infty: \quad \sqrt{-\bar{s}}\left[\bar{u}^{+}, \bar{\tau}^{+}\right] \rightarrow\left[u_{p}^{+}\left(y^{+}\right), y^{+}\right] .
\end{gathered}
$$


Here, the conditions for $y^{+}$large in (4.18b) are enforced by a balance of the pressure gradient with the inertia terms in $(4.18 a)$ on the basis of $(3.19 b)$, in order to allow for matching $u$ in the wall layer and the small-defect region. This behaviour is apparently incompatible with the balance of the pressure and shear stress gradients of the oncoming flow, as expressed by $(4.18 c)$. In order to resolve this inconsistency, one then has to consider in addition a limit where both $y^{+}$and $-\bar{s}$ are large and all terms in $(4.18 a)$ are of comparable order of magnitude. In this context, we mention that $u_{p}^{+} \sim y^{+} /(2 \kappa)$ as $y^{+} \rightarrow \infty$, by exploitation of (4.8), and taking into account that $\tau_{p}^{+}=y^{+}$. Then, this limit is characterised by $y^{+}=(-\bar{s})^{1 / 2} / \ln (-\bar{s})$ as $-\bar{s} \rightarrow \infty$ and its investigation is expected to shed light on the first breakdown and higher-order contributions to the upstream conditions $(4.18 c)$.

The problem (4.18) can be solved for $\bar{u}^{+}$in the range $-\infty<\bar{s}<0$, by means of downstream integration provided $\bar{\tau}_{t}^{+}$is modelled properly. However, the behaviour of $\left[\bar{u}^{+}, \bar{\tau}^{+}\right]$for $\bar{s} \rightarrow 0_{-}$, discussed extensively by Scheichl \& Kluwick (2008b), is crucial for the subsequent analysis rather than is the full solution. One finds that the flow is then essentially governed by inertia terms. Specifically, we obtain

$$
\begin{array}{ll}
\bar{s} \rightarrow 0_{-}: & {\left[\bar{u}^{+}, \bar{\tau}^{+}\right] \sim\left[\bar{u}_{0}^{+}, \bar{\tau}_{0}^{+}\right]\left(y^{+}\right)+(-\bar{s})^{1 / 6}\left[a_{-} \frac{\mathrm{d} \bar{u}^{+}}{\mathrm{d} y^{+}}, \bar{\tau}_{1}^{+}\left(y^{+}\right)\right]+O\left[(-\bar{s})^{1 / 2}\right],} \\
y^{+} \rightarrow 0: & \bar{u}_{0}^{+} \sim-\bar{b} y^{+3 / 2}+\cdots, \\
& a_{-}=48^{1 / 3} \Gamma\left(\frac{2}{3}\right) \doteq 4.9212, \quad \bar{b}=(4 / 3) \Gamma\left(\frac{1}{6}\right) / \Gamma\left(\frac{2}{3}\right) \doteq 5.4809 .
\end{array}
$$

Here $\bar{u}_{0}^{+}\left(y^{+}\right), \bar{\tau}_{0}^{+}\left(y^{+}\right)$depend on the particular form of $u_{p}^{+}\left(y^{+}\right)$in the upstream conditions $\left(4.18 c\right.$ ) (apart from the modelling of $\bar{\tau}_{t}^{+}$). We also note that $\bar{u}_{0}^{+}=o\left(1 / \ln y^{+}\right)$, as $y^{+} \rightarrow \infty$ due to $(4.18 b)$. Specifically, (4.19) follows from the investigation of a new adjustment region where $y^{+} /(-\bar{s})^{1 / 3}=O(1)$ and the viscous stress term plays a dominant role: there the flow is laminar-like as $u_{0}^{+} \sim y^{+2} / 2$, fitting to (3.19a), and the solution of (4.18) is predominantly unaffected by $\bar{\tau}_{t}^{+}$, according to (4.18b), and hence of universal self-similar character.

As a further finding of the analysis in that adjustment region, we have

$$
y^{+}=0, \quad \bar{s} \rightarrow 0_{-}: \quad \frac{\partial u^{+}}{\partial y^{+}} \sim 1-\frac{\bar{c} \bar{\pi}^{+}}{(-\bar{s})^{1 / 6}}+\cdots, \quad \bar{c}=\frac{\Gamma\left(\frac{1}{6}\right) \Gamma\left(\frac{1}{3}\right)}{3^{1 / 3} \pi^{1 / 2} \Gamma\left(\frac{2}{3}\right)} \doteq 4.3078 .
$$

The behaviour (4.20) indicates that the wall shear stress is reduced significantly and no longer determined by (3.16) and (3.24) for $\bar{s}=O\left(\bar{\pi}^{+6}\right)$. Even more importantly, a third (nonlinear) breakdown of the wall layer flow takes then place in the aforementioned adjustment region as $u^{+}$and $\bar{\pi}^{+} \bar{u}^{+}$become of the same order of magnitude. It is shown below that this scenario has to be considered from the viewpoint of viscous-inviscid interaction.

\section{The interaction process}

In the small-defect region of the boundary layer the wall-normal pressure gradient $\partial_{y} p$ becomes of the order of magnitude of the streamwise one, $\partial_{x} p$, when $-s$ decreases to $O(\delta)$. The conventional boundary layer approximation then ceases to be valid in the region of outer viscous-inviscid interaction in the vicinity of $\mathscr{D}$. However, a thorough investigation of this interaction mechanism has to account also for the flow at distances $y \ll \delta$ and thus requires knowledge of the near-wall flow and the associated scaling of the boundary layer. Therefore, its properties, associated with 


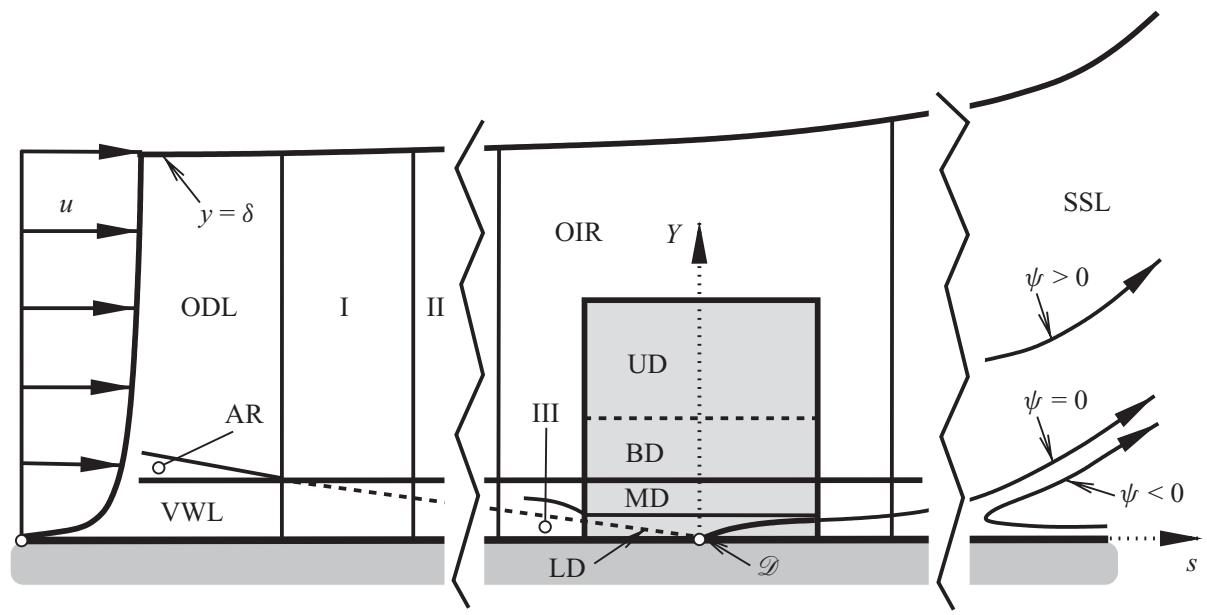

FiguRE 6. Asymptotic substructures of interactive boundary layer near $\mathscr{D}$ (not to scale, for caption see text): outer defect layer (ODL), viscous wall layer (VWL), adjustment region (AR) of $\S 4.1$ (the broken line indicates its virtual continuation towards $\mathscr{D}$ ), positions of first (I), second (II) and third (III) wall layer breakdowns, outer interaction region (OIR), separated shear layer (SSL), triple-deck (shaded), lower deck (LD), main deck (MD), buffer deck (BD), upper deck (UD).

the inner interaction process, are discussed next, while the outer interaction is topic of $\S 5.2$.

The therewith completed local subdivision of the interactive boundary layer flow is anticipated by the sketch in figure 6. Here the actual point of separation coincides with $\mathscr{D}$ for the sake of clarity.

\subsection{Inner interaction: internal triple-deck structure}

We begin by outlining the strategy adopted here to work out the triple-deck structure.

\subsubsection{Basic considerations}

One faces two key points in the analysis of the wall layer flow approaching $\mathscr{D}$ : first, the merging with the adjustment region, as addressed briefly in $\S 4.3 .1$ (first breakdown), and second the terminal structure of the non-interactive wall layer flow as $\bar{s} \rightarrow 0$, presented at the end of $\S 4.3 .2$ (second breakdown).

The first breakdown of the wall layer applies only on condition that $\delta \ll R e^{-1 / 3}$ or $\delta=O\left(R e^{-1 / 3}\right)$; if $\delta \gg R e^{-1 / 3}$, it is displaced by the occurrence of the outer interaction. In that case, the adjustment region is continued as a so-called blending or Reynolds stress sublayer located at the base of the interaction region and having a thickness measured by $\delta^{2}$, where the momentum equation $(2.2 a)$ still reduces to a balance between linearised convective terms and the turbulent effect $-\partial_{y}\left\langle u^{\prime} v^{\prime}\right\rangle$ to leading order. This situation is described by Melnik \& Chow (1975) in their study of the small-defect flow past the trailing edge of an inclined flat plate in a uniform stream. (Unfortunately, from the present point of view, their assumption of an incident fully developed turbulent boundary layer renders a self-consistent description of the wall layer flow passing the trailing edge an intractable task.) More generally, such a flow region is also found in other types of interacting fully developed turbulent boundary layers (see e.g. Melnik \& Chow 1975; Sykes 1980; Agrawal \& Messiter 1984). Therefore, determining the order of magnitude of $\delta$ in terms of $R e$ not only completes the scaling of the boundary layer but also answers the question of the 
existence of that blending layer, and in turn the asymptotic structure of the flow near $\mathscr{D}$.

This task is intrinsically tied to the second issue, which implies the formation of a further sublayer where the nonlinear inertia terms in (2.2a) are fully retained (cf. Scheichl \& Kluwick 2008b). As found from (3.15), the rescaled coordinates

$$
\bar{X}=\bar{s} / \bar{\pi}^{+6}, \quad \bar{Y}=y^{+} / \bar{\pi}^{+2}
$$

are quantities of $O(1)$. However, strong viscous-inviscid interaction of a boundarylayer-type flow in a small region encompassing both $\mathscr{D}$ and the actual point of separation, and thus dominated by the full nonlinear convective terms, is the sole possibility to regularise the B-V singularity within the limits of the Reynolds equations (2.2). This sublayer must serve as the active or lower deck in the expected triple-deck structure (cf. Neish \& Smith 1992; Rothmayer \& Smith 1998; Sychev et al. 1998). There the pressure gradient $\partial_{x} p$ is self-induced such that it both surmounts the $\mathrm{B}-\mathrm{V}$ singularity and avoids the formation of a Goldstein singularity. Henceforth, let

$$
\delta_{T D}=\bar{\delta} \bar{\pi}^{+6}, \quad \delta_{L D}=\delta_{v} \pi^{+2}
$$

define the triple-deck length scale and the thickness of the lower deck, respectively.

Consequently, for $\bar{X}=O(1)$, the core of the wall layer acts as the main deck, where the flow must also be of boundary layer type, i.e. the ratio of its thickness to the length of the sublayer in the $x$-direction is small. From (4.16), (4.17), (1.2), and the wall layer scaling given by (3.14) and (1.2), this ratio is found to be measured by $\epsilon^{8} \sigma^{7} R^{3}$ for $k=O(1)$, which yields the estimate $R e^{-1 / 2} \ll \sigma \ll R e^{-3 / 7}(\ln R e)^{8 / 7}$, in agreement with (3.24). Then $\delta \ll R e^{-1 / 3}$, which, as a first remarkable result here, precludes the existence of a blending layer.

\subsubsection{Lower deck and near-wall flow reversal}

Inspection of (3.14), (3.15) and (5.1) indicates that appropriate lower-deck expansions read

$$
\alpha \operatorname{Re\psi } \sim \bar{\pi}^{+4} \bar{\Psi}(\bar{X}, \bar{Y})+\cdots, \quad p-p_{0 \mathscr{D}} \sim \bar{\delta}^{1 / 2} \bar{\pi}^{+3} u_{0 \mathscr{D}}^{2} k P(\bar{X})+\cdots, \quad \bar{\pi}^{+} \ll 1 .
$$

Furthermore, the velocity gradient $\partial_{y^{+}} u^{+}=O(1)$ but $\tau_{t}^{+}=O\left(\bar{\pi}^{+6}\right)$, according to (3.18). Thus, (2.2a) and (2.3) give rise to the lower-deck problem for quasi-laminar separation in canonical form,

$$
\begin{gathered}
\partial_{\bar{Y}} \bar{\Psi} \partial_{\bar{Y} \bar{X}} \bar{\Psi}-\partial_{\bar{X}} \bar{\Psi} \partial_{\bar{Y}}^{2} \bar{\Psi}=-\mathrm{d} P / \mathrm{d} \bar{X}+\partial_{\bar{Y}}^{3} \bar{\Psi}, \\
\bar{Y}=0: \quad \bar{\Psi}=\partial_{\bar{Y}} \bar{\Psi}=0, \quad \bar{Y} \rightarrow \infty: \quad \bar{\Psi} / \bar{Z}^{2} \rightarrow 1 / 2, \quad \bar{Z}=\bar{Y}+A(\bar{X}), \\
\bar{X} \rightarrow-\infty: \quad \bar{\Psi} \rightarrow \bar{Y}^{2} / 2, \quad P /(-\bar{X})^{1 / 2} \rightarrow-2, \quad \bar{X} \rightarrow \infty: \quad P \rightarrow 0 .
\end{gathered}
$$

Once these equations are closed by the interaction law between the pressure and displacement functions $P(\bar{X})$ and $-A(\bar{X})$ (see $\S 5.1 .4$ ), they constitute the core problem governing the separation process. (The corresponding non-interactive problem considered by Scheichl \& Kluwick $2008 b$ is obtained formally by setting $P=-2(-\bar{X})^{1 / 2}$.)

The behaviour of $\bar{\Psi}$ for $\bar{Y} \rightarrow \infty$ is again triggered by the predominance of the inertia terms in $(5.3 a)$, in combination with the upstream conditions in $(5.3 c)$. A thorough examination of the non-interactive flow region far upstream where $\bar{Y} /(-\bar{X})^{1 / 3}=O(1)$ or, equivalently, $y^{+} /(-\bar{s})^{1 / 3}=O(1)$ recovers the universal laminar-type near-wall part of the terminal structure of the non-interactive wall layer flow and the associated 
nonlinear breakdown, as outlined in $\S 4$.3.2. We finally obtain

$$
\begin{aligned}
\bar{X} \rightarrow-\infty: & A \sim a_{-}(-\bar{X})^{1 / 6}+\cdots, \quad\left(\partial_{\bar{Y}}^{2} \bar{\Psi}\right)(\bar{X}, 0) \sim 1-\bar{c}(-\bar{X})^{-1 / 6}+\cdots, \\
\bar{Z} \rightarrow \infty: & \bar{\Psi} \sim \bar{Z}^{2} / 2-\bar{b} \bar{Z}^{3 / 2}+P(\bar{X})+O\left(\bar{Z}^{-3 / 2}\right),
\end{aligned}
$$

in agreement with (4.19) and (4.20). In the following, we demonstrate that the form of $\bar{\Psi}$ far downstream is fully determined by (5.3) when supplemented with an interaction law, provided gross separation takes place for some $\bar{X}=\bar{X}_{S}$ (where the rescaled wall shear stress $\left(\partial_{\bar{Y}}^{2} \bar{\Psi}\right)(\bar{X}, 0)$ changes sign). The expansion $(5.4 b)$ reflects the predominantly inviscid flow on top of the lower deck.

For $\bar{X} \gg 1$, the expansion $(5.4 b)$ apparently breaks down in the region of a mixing layer which encompasses the location of the separating streamline defined by $\bar{Y}=\bar{Y}_{S}(\bar{X})$, with $\bar{\Psi}\left(\bar{X}, \bar{Y}_{S}\right)=0$. This shear layer has an extent in the wall-normal direction of, say, $B(\bar{X})$ with $B=O(1)$ or larger, and $-B / A \ll 1$ as $-A \gg 1, \bar{X} \gg 1$, so that $\bar{\Psi} \sim B(\bar{X})^{2} \bar{F}(\bar{\eta}), \bar{\eta}=\bar{Z} / B=O(1)$. Inserting these last expressions into (5.3a) shows that the pressure gradient $\mathrm{d} P / \mathrm{d} \bar{X}$ is negligibly small there, and balancing inertia and viscous terms yields $B \sim \bar{X}^{1 / 3}$ and the mixing-layer problem as in the laminar counterpart of the separation problem, $\bar{F}^{\prime 2 / 3}-(2 / 3) \bar{F} \bar{F}^{\prime \prime}=\bar{F}^{\prime \prime \prime}$, together with the two conditions governed by the convective terms and compatible with the ambient flow, $\bar{F} \sim \bar{\eta}^{2} / 2$ as $\bar{\eta} \rightarrow \infty$ and $\bar{F}^{\prime}(-\infty)=0$, and $\bar{F}\left(\bar{\eta}_{S}\right)=0$. Here, $\bar{\eta}=\bar{\eta}_{S}$ defines the yet unknown position of the separating streamline which itself is regarded as part of the full solution of (5.3): $\bar{Y}_{S} \sim-A(\bar{X})+\bar{\eta}_{S} B(\bar{X})$. Therefore, $\bar{\eta}_{S}$ is arbitrary for the time being, as it is determined by a higher-order analysis. However, the solution $\bar{F}$ is unique when written in the form $\bar{F}\left(\bar{\eta}-\bar{\eta}_{S}\right)$, with $\bar{F}(-\infty)=-\bar{a} \doteq-1.2539$ (Neiland 1971; Stewartson \& Williams 1973; Diesperov 1984). Accordingly, for $\bar{Y}=O(A)$, we expect backflow that is governed by the inviscid form of $(5.3 a)$. Eliminating the pressure gives $\partial_{\bar{Y}}^{2} \bar{\Psi} \sim-\bar{\omega}(\bar{\Psi})$, where $\bar{\omega}$ denotes the vorticity. By matching $\bar{\Psi}$ with its representation holding in the mixing layer, we conclude that $\partial_{\bar{Y}}^{2} \bar{\Psi}=O\left(B^{2} / A^{2}\right)$; hence, $\bar{\omega}$ is treated as negligibly small. In turn, the slow irrotational reversed flow in the bulk of the lower deck is found to be described by $\bar{\Psi} \sim \bar{a} \bar{X}^{2 / 3} \bar{Y} / A(\bar{X})<0, P \sim-\bar{a}^{2} \bar{X}^{4 / 3} /\left(2 A^{2}\right)$, $\bar{X} \gg 1$, where $A$ varies basically algebraically with $\bar{X}$. Finally, a sublayer emerges for $\bar{Y}=O\left[\bar{X}^{1 / 6}(-A)^{1 / 2}\right]$ where all terms in $(5.3 a)$ are retained to leading order, governing viscous self-preserving reversed flow.

Therefore, even without knowing in advance the behaviour of $A(\bar{X})$ for $\bar{X} \rightarrow \infty$ in detail, we end up with a three-tiered structure of the downstream flow as in the laminar case, where $A=O\left(\bar{X}^{3 / 2}\right)$, as discussed elaborately by Sychev (1972) and Sychev et al. (1998), in particular.

\subsubsection{Main deck}

The expansion (5.4b) allows a match with the flow in the bulk of the wall layer for $\bar{X}$ of $O(1)$. There the leading-order stress balance stays intact and the corresponding expansions (taken directly from the non-interactive analysis; see Scheichl \& Kluwick $2008 b)$ are

$$
\begin{aligned}
\alpha \operatorname{Re} \psi & \sim \int_{0}^{y^{+}}\left[u_{0}^{+}(t)+\bar{\pi}^{+} \bar{u}_{0}^{+}(t)\right] \mathrm{d} t+\bar{\pi}^{+2} A(\bar{X}) u_{0}^{+}\left(y^{+}\right)+O\left(\bar{\pi}^{+3}\right), \\
p-p_{0 \mathscr{D}} & \sim \bar{\delta}^{1 / 2} \bar{\pi}^{+3} u_{0 \mathscr{D}}^{2} k P(\bar{X})+\cdots,
\end{aligned}
$$

supplemented by $-\left\langle u^{\prime} v^{\prime}\right\rangle \sim \alpha \epsilon^{2} u_{0 \mathscr{T}}^{2} \tau_{0}^{+}\left(y^{+}\right)+\cdots$. The expansion (5.5a) in combination with $(5.4 a)$ matches $(4.19 a)$. We remark that the logarithmic law of the wall is still present, according to $(3.19 b)$. 
The separating streamline, originating in the lower deck, penetrates into the core of the wall layer far downstream, where $\bar{Y} \sim A=O\left(1 / \bar{\pi}^{+2}\right)$. There the forms of the wall functions $u_{0}^{+}\left(y^{+}\right), \tau_{0}^{+}\left(y^{+}\right)$are finally modified substantially.

\subsubsection{Upper deck and boundary layer scaling}

In an ad hoc analysis assuming non-interactive flow (i.e. for $P=-2(-\bar{X})^{1 / 2}$ and $A(\bar{X})$ known from the solution of $(5.3))$, the expansions $(5.5 a, b)$ provide a match with the flow in the small-defect region (cf. Scheichl \& Kluwick 2008b). In striking contrast, here the flow structure on top of the wall layer is crucial for the determination of the interaction law. Since the flow is there predominantly governed by the equations for inviscid flow, we have to deal specifically with a square region having an extent measured by $O\left(\delta_{T D}\right)$, so that here $\hat{Y}=y / \delta_{T D}=O(1)$.

The B-V singularity can only be avoided by the flow in the upper deck if the first $\bar{X}$-dependent term in the expansion of $\psi$ in that flow region accounts for the leadingorder variations of both the pressure and the displacement, exerted by the lower deck and transferred unchanged through the main deck. We thus conclude that this expansion starts with terms 'frozen' at $x=x_{\mathscr{D}}$, known from the non-interactive flow analysis of the small-defect region (rewritten in terms of $\hat{Y}$ and accordingly expanded), and is followed by the $\bar{X}$-dependent contribution arising from the displacement of the lower deck and accounting for the mechanism of inner interaction. We, therefore, by inspection of $(3.19 b),(3.14)$ and (5.2) write

$$
\begin{aligned}
\psi-\delta_{T D} u_{0 \mathscr{D}} \hat{Y}+\cdots & \sim(\alpha \operatorname{Re})^{-1} \bar{\pi}^{+2} \ln \left(\sigma R e / \delta_{T D}\right) \kappa^{-1} \Psi_{T D}(\bar{X}, \hat{Y})+\cdots, \\
p-p_{0 \mathscr{D}} & \sim \bar{\delta}^{1 / 2} \bar{\pi}^{+3} u_{0 \mathscr{D}}^{2} k P_{T D}(\bar{X}, \hat{Y})+\cdots .
\end{aligned}
$$

Herein, $\Psi_{T D}$ and $P_{T D}$ are governed by the linearised Euler equations that the equations of motion (2.2) reduce to in the approximation considered. This requires the ratio of the coefficients of $\Psi_{T D}$ and $P_{T D}$ in (5.6) to be proportional to $\delta_{T D} /\left(\Gamma u_{0 \mathscr{D}}\right)$, with $\Gamma$ denoting a similarity parameter assumed to be $O(1)$. Since the terms on the left-hand side of $(5.10 a)$ due to the incident flow 'frozen' at $x=x_{\mathscr{D}}$ are independent of $\bar{X},(2.2)$ then reduces to

$$
\left[\partial_{\hat{Y}},-\partial_{\bar{X}}\right] \partial_{\bar{X}} \Psi_{T D}=-\Gamma\left[\partial_{\bar{X}}, \partial_{\hat{Y}}\right] P_{T D}
$$

We furthermore obtain with the aid of (1.2), (4.16), (4.17), (5.1b) and (3.24) in the regime (1.1) the relationships

$$
\bar{\chi} \sim \bar{K}\left(\frac{\kappa R e}{\epsilon^{10} \ln \bar{\chi}}\right)^{1 / 9}, \quad \bar{\chi}=\sigma R e^{5 / 9} \gg 1, \quad \bar{K}=\left(\frac{k^{8}}{9 \Gamma u_{0 \mathscr{D}}^{4}}\right)^{1 / 9}=O(1) .
$$

From (5.8), the desired scaling of the interactive boundary layer is found in terms of

$$
\begin{gathered}
\epsilon \sim 9 \kappa / \ln R e, \quad \sigma=\bar{K} R e^{-4 / 9} / \epsilon, \\
\delta_{v}=O\left(\epsilon R e^{-5 / 9}\right), \quad \delta_{T D}=O\left(R e^{-4 / 9}\right), \quad \delta_{L D}=O\left(R e^{-6 / 9}\right), \\
\bar{\delta}=O\left(\epsilon^{3} R e^{-1 / 9}\right) .
\end{gathered}
$$

The relationships (5.9a) describe slightly underdeveloped turbulent flow according to (3.27), where we end up with $\chi=\bar{K} / \epsilon$ and $\mu=1 / 18$. Also, we find that $I_{t}=\bar{K} R e^{-4 / 9}$ and $\alpha=\bar{K}(9 \kappa)^{-2}(\ln R e)^{2} R e^{-4 / 9}$ by $(1.2)$, which gives $T=O\left[(\ln R e)^{2} R e^{1 / 18}\right]$ by $(3.25 a)$ and finally confirms $(3.25 b)$. The length scales quoted in $(5.9 b)$ follow accordingly from (3.14) and (5.1b), with the help of (4.17), and basically agree with those suggested by Neish \& Smith (1992). We note, however, that according to the more detailed analysis 
carried out here, the upper-deck scale $\delta_{T D}$ is smaller than the boundary layer scale $\delta$ by a factor $\epsilon$. This has two remarkable consequences: first, it means that the triple-deck scale is slightly smaller than in laminar case, where it is $O\left(R e^{-3 / 8}\right)$ (cf. Rothmayer \& Smith 1998; Sychev et al. 1998), and second the triple-deck structure is 'squashed' in the square region with an extent measured by $\delta$, which renders the notions of inner and outer interaction sensible. We finally note that $\delta^{+}$defined by (3.26) varies with $\epsilon^{2} R e^{-1 / 9}$, and $(5.9 c)$ is a consequence of (4.16). Thus, the second breakdown of the wall layer flow occurs upstream of the onset of the outer interaction.

As a result of the 'frozen' state of the boundary layer expressed by (4.1) and (3.21a), one finds the vorticity in the upper deck to vary as $-\epsilon u_{0 \mathscr{D}} \kappa^{-1} /\left(\delta_{T D} \hat{Y}\right)+\cdots$, which is $O(1 / \sigma)$. Also, the flow in the upper deck is still of small-defect form and hence matches that in the region of the outer interaction identically. In order to clarify this situation further in view of the scalings $(5.9 a)$ with $\bar{K}$ given by (5.8), we now re-establish the upper-deck expansions (5.6) by using (5.1b) and (4.17) as

$$
\begin{aligned}
\psi / u_{0 \mathscr{D}}-\delta_{T D} \hat{Y}+\cdots & \sim \delta_{T D}^{3 / 2} k\left[\Psi_{T D}(\bar{X}, \hat{Y}) / \Gamma+O(\epsilon)\right]+\cdots, \\
\left(p-p_{0 \mathscr{D}}\right) / u_{0 \mathscr{D}}^{2} & \sim \delta_{T D}^{1 / 2} k\left[P_{T D}(\bar{X}, \hat{Y})+O(\epsilon)\right]+\cdots .
\end{aligned}
$$

Here, the terms $O(\epsilon)$ in square brackets arise from $(5.5 a)$ subject to the logarithmic behaviour $(3.19 b)$.

It is noted that matching of the gradients with respect to $y$ requires the introduction of a so-called buffer deck, located between the main and upper decks, as a consequence of $(5.5 a)$ and $(3.19 b)$. Comparison of the latter expansion with $(5.10 a)$ shows that $\ln \operatorname{Re} \hat{Y} \partial_{\hat{Y}} \Psi_{T D}$ is of the same order of magnitude as $y^{+} \mathrm{d} u_{0}^{+} / \mathrm{d} y^{+}$in this flow region. By taking notice of $(5.11 b)$, one immediately infers that this additional layer is located at $\hat{y}=\hat{Y} / \epsilon=O(1)$. This indicates that the ratio of its thickness to that of the upper deck is again given by $\epsilon$. However, this buffer deck behaves fully passively as the expansions of the flow quantities holding there are essentially found by re-expanding their counterparts in the adjacent layers when rewritten in terms of $\hat{y}$. We therefore refrain from considering this region in more detail.

Elimination of the pressure $P_{T D}$ in (5.7) yields Laplace's equation in Cartesian coordinates,

$$
\bar{\nabla}_{c}^{2} \Psi_{T D}=0, \quad \bar{\nabla}_{c}^{2}=\delta_{T D}^{2} \nabla_{p}^{2}=\partial_{\bar{X}}^{2}+\partial_{\hat{Y}}^{2},
$$

in agreement with (3.5), (A 7) and (2.2c), as a possible $\bar{X}$-independent 'constant' of integration is conveniently absorbed in the 'frozen'-flow contribution to $(5.10 a)$. By matching (5.10) and (5.5), we find that

$$
\Psi_{T D}(\bar{X}, 0)=A(\bar{X}), \quad \partial_{\hat{Y}} \Psi_{T D}(\bar{X}, 0)=-\Gamma P(\bar{X}), \quad P(\bar{X})=P_{T D}(\bar{X}, 0),
$$

and deduce that $\Psi_{T D} \sim A(\bar{X})-\Gamma P(\bar{X}) \hat{Y}$ for $|\bar{X}| \rightarrow \infty, \hat{Y}=O(1)$. A standard investigation of the Laplacian subject to the conditions for $P, A$ in $(5.3 c),(5.4 a)$ and the form of the downstream decay of $P$ elucidated at the end of $\S 5.1 .2$ then suggests

$$
\begin{aligned}
\bar{R}=\frac{r}{\delta_{T D}}=\sqrt{\bar{X}^{2}+\hat{Y}^{2}} \rightarrow \infty: \Psi_{T D} \sim \frac{\Gamma}{k} \psi_{0,3 / 2}(\bar{R}, \theta)+\Lambda \bar{R}^{1 / 2} \cos (\theta / 2) \\
+\left(2 a_{-} / \sqrt{3}\right) \bar{R}^{1 / 6} \cos (\theta / 6)+O(\ln \bar{R}) .
\end{aligned}
$$

Here $r, \theta$, and $\psi_{0,3 / 2}$ are defined by (3.5) and (3.6b), respectively. The expansion (5.11) was earlier established by Brown \& Stewartson (1970) and Melnik \& Chow (1975) in their study of laminar non-symmetric flow past a sharp trailing edge, where the external flow exhibits a B-V-like singularity and thus an upper deck having 
alike properties. They demonstrated that the dominant eigensolution of Laplace's equation proportional to the constant $\Lambda$, unknown at this stage, indeed varies strictly algebraically with $\bar{R}^{1 / 2}$, in order to match the upstream behaviour of the lower-deck solution governed by (5.3) and (5.4). The expansion (5.11c) closes the upper-deck problem (5.11), based on potential flow theory and being fully equivalent to that in the laminar case (cf. Sychev et al. 1998).

The higher-order contributions in the far-field behaviour (5.11c) allow corresponding estimates of $A(\bar{X})$ and $P(\bar{X})$, more accurate than those given in $(5.3 c)$ and $(5.4 a)$ as necessary for later considerations. We obtain

$$
\begin{aligned}
\bar{X} \rightarrow-\infty: & A \sim a_{-}(-\bar{X})^{1 / 6}+O(\ln (-\bar{X})), \\
& P \sim-2(-\bar{X})^{1 / 2}-\frac{\Lambda}{2 \Gamma}(-\bar{X})^{-1 / 2}-\frac{a_{-}}{6 \sqrt{3} \Gamma}(-\bar{X})^{-5 / 6}+\cdots, \\
\bar{X} \rightarrow \infty: \quad & A \sim-a_{+} \bar{X}^{3 / 2}+\Lambda \bar{X}^{1 / 2}+\frac{2 a_{-}}{\sqrt{3}} \bar{X}^{1 / 6}+O(\ln \bar{X}), \quad a_{+}=\frac{4 \Gamma}{3}, \\
& P \sim-p_{+} \bar{X}^{-5 / 3}+\cdots, \quad p_{+}=\left(\bar{a} / a_{+}\right)^{2} / 2 .
\end{aligned}
$$

The relationship (5.12d) attests to a structure of the early-stage reversed flow, having its origin in the lower deck as outlined in $\S 5.1 .2$, known from the laminar counterpart. In the case considered here, the free streamline penetrates into the bulk of the viscous wall layer for $\delta \ll s=O\left(\epsilon^{2 / 3} R e^{-10 / 27}\right)$, as commented in $\S 5.1 .3,(5.12 c)$, (5.1a) and (4.17). Therefore, the wall function $u_{0}^{+}\left(y^{+}\right)$and the leading-order stress balance in the wall layer, reading $\tau_{0}^{+}=1$, remain unaltered even for $s=O(\delta)$, the length scale characteristic of the outer interaction.

Equation (5.7) states that $\Gamma P_{T D},-\partial_{\bar{X}} \Psi_{T D}$ represent a harmonic conjugate pair. An analogous conclusion holds for the derivatives of the function $\Psi_{T D}-(\Gamma / k) \psi_{0,3 / 2}$, harmonic in the upper half-plane $\hat{Y} \geqslant 0$ and subject to $(5.11 b)$. Then,

$$
\bar{P}=\Gamma P+2 \Gamma H(-\bar{X})(-\bar{X})^{1 / 2}, \quad-\bar{A}^{\prime}=-\mathrm{d} A / \mathrm{d} \bar{X}-2 \Gamma H(\bar{X}) \bar{X}^{1 / 2},
$$

with $H$ denoting the Heaviside unit step function, form a Hilbert pair,

$$
\left[\bar{P},-\bar{A}^{\prime}\right](\bar{X})=\frac{1}{\pi} f_{-\infty}^{\infty} \frac{\left[\bar{A}^{\prime}, \bar{P}\right](S)}{\bar{X}-S} \mathrm{~d} S .
$$

As an important aspect, (5.13) guarantees the downstream decay of $P$ originally required by $(5.3 c)$. Finally, these relationships, together with the dominant behaviour of $P$ far upstream, determine that of $A$ far downstream, see (5.12c), and thus the structure of the post-separated flow according to the analysis of $\S 5.1 .2$. The resulting interaction law (5.13) closes the lower-deck problem (5.3), then forming a triple-deck problem, parametrised by $\Gamma$, which is formally identical with that found in the theory of laminar separation, as suggested for the turbulent case by Neish \& Smith (1992). Smith (1977) demonstrated by a numerical investigation that a (unique) solution to this triple-deck problem only exists for a specific (positive) eigenvalue of $\Gamma$; refined solutions were obtained by Korolev (1980) and van Dommelen \& Shen (1984). This problem can be cast into standard form (cf. Sychev et al. 1998) by means of the affine transformation $\bar{X} \mapsto \bar{X} / \bar{\Gamma}^{6}, \hat{Y} \mapsto \hat{Y} / \bar{\Gamma}^{2}, \bar{\Psi} \mapsto \bar{\Psi} / \bar{\Gamma}^{4}$ (i.e. $A \mapsto A / \bar{\Gamma}^{2}$ ), $P \mapsto P / \bar{\Gamma}^{4}$, with

$$
2 \Gamma^{1 / 8}=2 \bar{\Gamma} \doteq 0.415 \pm 0.005
$$

as the currently most accurate figure of this eigenvalue given by van Dommelen \& Shen (1984). This situation renders the scalings (5.9) and, notably, the value of $\Lambda$ 
uniquely determined for a given value of the external-flow parameter $k$. The far-field eigensolution of Laplace's equation, proportional to $\Lambda$ as seen from $(5.11 c),(5.12 b)$ and $(5.12 c)$, reflects the invariance of the triple-deck solution with respect to an arbitrary shift of the origin $\bar{X}=0$ for $|\bar{X}|$ large. In contrast to the laminar case, here $\Lambda$ proves crucial in connection with matching the 'inner' and 'outer' interacting flows as shown next.

\subsection{Outer interaction and separation criterion}

We introduce appropriately stretched variables $[R, X, Y, \Psi]=\left[r, s, y, \psi / u_{0 \mathscr{D}}\right] / \delta_{\mathscr{D}}$, which are assumed to be $O(1)$ in the square region of the outer interaction, forming the continuation of the main tier of the boundary layer and continued as a separated shear layer further downstream. Here $R=\left(X^{2}+Y^{2}\right)^{1 / 2}$ and $\delta_{\mathscr{D}}$ denotes the value of the boundary layer thickness for $s=0$, expanded as $\delta_{\mathscr{D}} \sim \sigma\left[\Delta_{\mathscr{D}}+O(\epsilon)\right]$ according to (3.13a) and (4.1). It is convenient to write

$$
\delta_{T D} / \delta_{\mathscr{D}} \sim(\epsilon / K)[1+O(\epsilon)], \quad K=k^{2} \Delta_{\mathscr{D}}(k) /(9 \Gamma)=O(1),
$$

which follows after some manipulations from (5.1b), (4.17), (5.8) and (5.9a).

In the asymptotic limit currently under focus, separation is seen to take place at the origin $R=0$, which then forms a singular point in the flow description. Moreover, in the viscous wall layer, expansions of the type (5.5a) hold,

$$
\begin{gathered}
\alpha \operatorname{Re\psi } \sim \int_{0}^{y^{+}} u_{0}^{+}(t) \mathrm{d} t+\cdots+\bar{\pi}^{+2} u_{0}^{+}\left(y^{+}\right)\left\{\begin{array}{cc}
a_{-}(-K X / \epsilon)^{1 / 6}+\cdots, & X<0, \\
-a_{+}(K X / \epsilon)^{3 / 2}+\cdots, & X>0,
\end{array}\right. \\
p-p_{0 \mathscr{D}} \sim-\delta_{\mathscr{D}}^{1 / 2} u_{0 \mathscr{D}}^{2} k\left\{\begin{array}{l}
2(-X)^{1 / 2}+\cdots, \\
p_{+}(\epsilon / K)^{13 / 6} X^{-5 / 3}+\cdots,
\end{array} \quad X>0,\right.
\end{gathered}
$$

in agreement with (5.12). In turn, matching the streamfunction with its representations in the wall layer for $X \neq 0$, the upper deck for $R \rightarrow 0$ in the form (5.11c), the external flow as given by (3.1) and (3.6), and, eventually, the oncoming boundary layer as expressed by (3.13), (4.1) and (3.7b), and taking note of the dominance of inertia terms in (2.2) in the region of the outer interaction suggests the expansions

$$
\begin{aligned}
\Psi \sim & +\delta_{\mathscr{D}}^{1 / 2} \Psi_{O P}(X, Y ; k)+O\left(\delta_{\mathscr{D}}\right)-\epsilon\left[F_{\mathscr{D}}(Y ; k)\right. \\
& \left.-\delta_{\mathscr{D}}^{1 / 2} \Psi_{O I}(X, Y ; k)+O\left(\delta_{\mathscr{D}}\right)\right]+O\left(\epsilon^{2}\right), \\
\delta / \delta_{\mathscr{D}} & \sim 1+\delta_{\mathscr{D}}^{1 / 2}\left[D_{1 / 2}(X ; k)+O(\epsilon)\right]+O\left(\delta_{\mathscr{D}}\right) .
\end{aligned}
$$

That is, effects of surface curvature are insignificant for the outer interaction process, and the small-defect structure is preserved as $F_{\mathscr{D}}(Y ; k)$ represents the locally 'frozen' state of the oncoming boundary layer: convective terms in (2.2) are linearised about the unperturbed velocity expressed by $[u, v]=\left[u_{0 \mathscr{D}}, 0\right]$, and the leading-order boundary layer velocity profile given by $F_{\mathscr{O}}^{\prime}$ is transferred unchanged into the separated shear layer, forming for large values of $X$. We remark that the Reynolds shear and normal stress gradients first enter the equations that govern contributions of $O\left(\epsilon \delta_{\mathscr{O}}\right)$ in (5.18), in the form of inhomogeneities.

The equations governing $\Psi_{O P}$ and $\Psi_{O I}$ are derived from (2.2) by elimination of the pressure in straightforward manner as in $\$ 5.1 .4$. One then finds in leading order the potential flow problem

$$
\begin{gathered}
\nabla_{c}^{2} \Psi_{O P}=0, \quad \nabla_{c}^{2}=\delta_{\mathscr{D}}^{2} \nabla_{p}^{2}=\partial_{X}^{2}+\partial_{Y}^{2}, \\
X<0: \quad \Psi_{O P}(X, 0 ; k)=0, \quad X>0: \quad \partial_{Y} \Psi_{O P}(X, 0 ; k)=0,
\end{gathered}
$$




$$
R \rightarrow 0: \quad\left|\Psi_{O P}\right| / R^{3 / 2}<\infty, \quad R \rightarrow \infty: \quad \Psi_{O P} \sim \psi_{0,3 / 2}(R, \theta ; k),
$$

where we refer to (3.5), (A 7) and (3.6b). The condition for $R \rightarrow 0$ in (5.19c) asserts that a singularity at the separation point $R=0$ stronger than the original $\mathrm{B}-\mathrm{V}$ singularity is unacceptable in a self-consistent interactive-flow description. By exploiting the extremal properties of solutions of Laplace's equation or applying the Mellin transform, one can show that

$$
\Psi_{O P} \equiv \psi_{0,3 / 2}(R, \theta ; k)=(2 \sqrt{2} k / 3) \breve{\Psi}, \quad \breve{\Psi}(X, Y)=(R-2 X) \sqrt{R+X},
$$

is the only conceivable solution of (5.19). This not only confirms (5.16) and agrees with $(5.11 c)$ by matching but also a posteriori justifies the strategy followed in $\S 5.1$, namely, that the B-V singularity is effectively avoided by means of the inner interaction mechanism.

As a crucial result, the contribution $O\left(\epsilon \delta_{\mathscr{D}}^{1 / 2}\right)$ to the expansion (5.18) describes an induced vortex flow that accounts for the interaction of the boundary layer flow with the irrotational leading-order disturbance $O\left(\delta_{\mathscr{D}}^{1 / 2}\right)$. Here, the vorticity transport equation reduces to Poisson's equation,

$$
\nabla_{c}^{2} \Psi_{O I}=-F_{\mathscr{D}}^{\prime \prime \prime}(Y ; k) \Psi_{O P}(X, Y ; k)
$$

Now, primes denote derivatives with respect to $Y$. The right-hand side of $(5.21 a)$ represents the negative $X$-dependent leading-order contribution of the vorticity generated in the boundary layer upstream and convected with the irrotational flow. Here a further $X$-independent adding term that results from integration in the derivation of $(5.21 a)$ has been set to zero as it would imply perturbations $O\left(\sigma^{1 / 2}\right)$ in (3.13a) and, thus, unacceptably alter the original structure of the incident (attached) boundary layer flow. Also, we have

$$
\begin{gathered}
X<0: \Psi_{O I}(X, 0 ; k)=0, \quad X>0, \quad Y \rightarrow 0: \quad \partial_{Y} \Psi_{O I}+F_{\mathscr{D}}^{\prime \prime} \Psi_{O P} \rightarrow 0 \\
X \rightarrow-\infty: \Psi_{O I} \sim 2 k \sqrt{-X} G_{\mathscr{D}}(Y ; k), \quad G_{\mathscr{D}}=2 F_{\mathscr{D}}-Y F_{\mathscr{D}}^{\prime}, \\
Y=1: \partial_{Y} \Psi_{O I}=0 .
\end{gathered}
$$

The conditions $(5.21 b)$ arise from matching the streamfunction and the pressure in the outer and the wall layer, according to (5.16). The requirements of matching with the oncoming boundary layer (see (4.1), (4.2) and (4.4)) lead to (5.21c), as $y \sim \eta\left[1-2 k(-s)^{1 / 2}\right]$ as $s \rightarrow 0_{-}$, where the near-wall form of $F_{\mathscr{D}}(Y ; k)$ is given by $(4.6 a)$ and (4.10). Here, we add that $\Psi_{O I}$ is obviously subject to subexponential growth for $X \rightarrow \infty$. Furthermore, $(5.21 d)$ is due to patching the solution with the disturbance $O(\epsilon \delta)$ in the ambient external flow, as given by (3.1) for $X=O(1), y=O(1)$. Finally, an investigation of $(5.21 a)$ subject to $(5.21 b)$ gives

$$
\pi>\theta>0, \quad R \rightarrow 0: \quad \Psi_{O I} \sim E(k) R^{1 / 2} \cos (\theta / 2)+\cdots, \quad E=9 \Lambda /\left[k \Delta_{\mathscr{D}}(k)\right],
$$

where the specific form of the coefficient $E$ is required by matching the outer and inner interacting flows by using $(5.10 a),(5.11 c)$ and (5.17), with the aid of (5.15).

Apart from the matching conditions (5.21b) for $X>0$ and (5.22), the description of the outer interaction closely resembles that in a fully developed turbulent trailingedge flow as put forward by Melnik \& Chow (1975, pp. 224-225, with (5.21a) having the wrong sign on its right-hand side). However, the presence of a wall further downstream, as in the problem considered here, leads to a change in detail and makes the treatment more involved. This becomes evident by evaluation of $(5.21 a)$ subject to $(5.21 b)$ and supplemented with (5.20), which reveals a contribution adding to the logarithmic portion of the initial velocity profile $F_{\mathscr{D}}^{\prime}(Y ; k)$ upstream of separation, 




FigURE 7. Boundary layer thickness near separation in normalised form (for labelling see text).

superseded by a stronger singular behaviour immediately downstream as for

$$
Y \rightarrow 0: \Psi_{O I} \sim-\frac{2 k}{3 \kappa} \begin{cases}3(-X)^{1 / 2} Y \ln Y+O(Y), & X<0, \\ 2 X^{3 / 2} \ln Y+O(1), & X>0 .\end{cases}
$$

The analysis of the interaction process described so far is unaffected by any turbulence closure. This enables us to also express the local variation of the boundary layer edge represented by $D_{1 / 2}$ in (5.18) in closed form. It is found by patching the vorticity expressed as $-\nabla^{2} \psi \sim-\left(u_{0 \mathscr{D}} / \delta_{\mathscr{D}}\right) \nabla_{c}^{2} \Psi$ for $y=\delta$. There it must vanish up to and including the orders quoted explicitly in (5.17), which yields $D_{1 / 2}=-\Psi_{O P}(X, 1 ; k)$ by $(5.21 a)$ (since $\left.F_{\mathscr{D}}^{\prime \prime \prime}(1 ; k)>0\right)$. This result matches the variation of $\delta$ in the oncoming flow as expressed by $(3.13 a)$, (4.1) and (4.2), as $D_{1 / 2} \sim-2 k(-X)^{1 / 2}+\cdots, X \rightarrow-\infty$, and gives $D_{1 / 2} \sim(4 k / 3) X^{3 / 2}+\cdots, X \rightarrow \infty$. The latter expression confirms that the region of outer interaction recovers as a separated shear layer which coincides with the region of breakdown along $\mathscr{S}$ for $s \rightarrow 0_{+}$, addressed briefly in connection with $(3.9 b)$. We remark that this local variation of the boundary layer thickness is already indicated in figure 6 . Its canonical representation $\breve{D}(X)=-\breve{\Psi}(X, 1)$ inferred from (5.20) is displayed in figure 7, together with the asymptotes for $|X|$ being large as noted above.

A particular solution of the elliptic problem posed by (5.21) can be constructed through exploitation of standard methods. Hence, we differentiate (5.21a) triply with respect to $X$, in order to cope with the growth of the inhomogeneity for $|X| \rightarrow \infty$ resulting from (5.20), which gives in the limit

$$
\varepsilon=\frac{Y}{X} \rightarrow 0: \quad \Psi_{O P} \sim 2 k|X|^{1 / 2} \begin{cases}Y\left[1+\varepsilon^{2} / 24+O\left(\varepsilon^{4}\right)\right], & X \rightarrow-\infty, \\ (2 X / 3)\left[-1+3 \varepsilon^{2} / 8+O\left(\varepsilon^{4}\right)\right], & X \rightarrow \infty .\end{cases}
$$

By writing $\Psi_{O I}(X, Y ; k)=\Psi_{O I h}+\Psi_{O I}$, we then find

$$
\begin{aligned}
\Psi_{O I p}(X, Y ; k)= & \int_{-\infty}^{X}\left[\int_{-\infty}^{S_{1}} \int_{-\infty}^{S_{2}} \Phi\left(S_{3}, Y ; k\right) \mathrm{d} S_{3} \mathrm{~d} S_{2}+k \frac{H\left(-S_{1}\right)}{\sqrt{-S_{1}}} G_{\mathscr{D}}(Y ; k)\right] \mathrm{d} S_{1} \\
& +2 k H(-X) \sqrt{-X} G_{\mathscr{D}}(Y ; k), \\
\Phi(X, Y ; k)= & -\frac{1}{2 \pi} \int_{-1}^{1} \operatorname{sgn}(Z) F_{\mathscr{D}}^{\prime \prime \prime}(|Z| ; k) \int_{-\infty}^{\infty} \Pi(S,|Z| ; k) \ln R^{\star} \mathrm{d} S \mathrm{~d} Z
\end{aligned}
$$




$$
\Pi(X, Y ; k)=\partial_{X}^{3} \Psi_{O P}=-3 \Psi_{O P} /\left(8 R^{3}\right), \quad R^{\star}=\sqrt{(X-S)^{2}+(Y-Z)^{2}},
$$

for a particular solution of (5.21a), which satisfies (5.21b) and (5.21c) rather than (5.21d) and (5.22). The determination of a complementary non-trivial homogeneous solution $\Psi_{O I h}(X, Y ; k)$ which supplements (5.25) to yield a (unique) composite solution of (5.21) is critical for an advanced flow description, and efforts to solve (5.21) are under way.

Eventually, the procedure of fixing the value of the external-flow parameter $k$, i.e. the position $x=x_{\mathscr{D}}(k)$ of inviscid flow detachment, on the basis of a rationally derived separation criterion for turbulent flow is delineated by the following four steps:

(a) extract the (non-vanishing) value of the coefficient $\Lambda$ in the second-order term of $(5.12 c)$ from the unique eigensolution of the triple-deck problem;

(b) seek a formal solution of the well-posed problem (5.21) in dependence of $k$;

(c) compute the quantity $E$ in (5.22), either (semi-)analytically or numerically;

(d) evaluate the resulting solvability condition for (5.21),

$$
9 \Lambda=k \mathscr{F}_{x=0}^{x=x_{\mathscr{P}}}\left\{U_{\mathscr{S}}(x ; k)\right\}, \quad \mathscr{F}=\Delta_{\mathscr{D}} E,
$$

representing the desired separation criterion.

It is insinuated in (5.26) that $\mathscr{F}$ represents a functional of the surface speed $U_{\mathscr{S}}$ that drives the boundary layer from stagnation towards separation. Here we point to the dependences of the flow variables $\Psi_{O P}, \Psi_{O I}$ on $k$ and the 'frozen' state of the boundary layer entering the right-hand side of $(5.21 a)$. Thus, (5.26) accounts for the global external flow as well as the upstream history of the boundary layer, as one would expect from a separation criterion that deals with the case $k=O(1)$, though deduced from a local asymptotic analysis. It is intriguing how this is achieved by matching due to the universal structure of the triple-deck solution.

The separation criterion (5.26) completes the description of the entire process of turbulent separation to the leading approximation.

\section{Conclusions and further outlook}

The comprehensive description of the time-mean bluff-body separation presented here appears to be self-consistent and physically relevant when the global Reynolds number, $R e$, is so large that the potential-flow parameter $k$ is independent of $R e$. In this situation, separation is provoked at a distance from the leading edge of the body that is comparable to its typical dimension $\tilde{L}$. It is significant that the asymptotic picture of the initially attached boundary layer is founded on a minimum of assumptions merely regarding the scaling of the turbulent motion. These consequently determine the flow structure in a region centred around the point of separation with an extent comparable to the boundary layer thickness $\delta$. It is interesting that the scaling properties of the boundary layer cannot be determined entirely unless the process of viscous-inviscid interaction that governs separation is taken into account. We further emphasize that the major results of the analysis are qualitatively unaffected by a specific turbulent shear stress closure provided that it satisfies restrictions which reflect the asymptotic properties of the overlap of the predominantly turbulent region of the boundary layer and the viscous wall layer. Effectively, classical mixing-length-based arguments suffice to disclose all the essential features of the separating flow. Altogether, the theory is essentially based upon the following premises.

(i) The body has a perfectly impermeable rigid smooth surface.

(ii) The flow is nominally two-dimensional. 
(iii) The flow on the body scale in the high-Reynolds-number limit is described by the Euler equations.

(iv) Free-stream turbulence is of no importance.

(v) All components of the Reynolds stress tensor are of comparable magnitude.

(vi) Each subregion of the turbulent boundary layer is characterised by a single velocity scale.

(vii) The wall layer is in equilibrium (initially firmly attached turbulent boundary layer).

We find that a rational description of separation starting with the classical picture of an initially attached fully developed two-tiered turbulent boundary layer is affected by two key aspects, depending on whether the adverse pressure gradient near separation exerted by the external flow is bounded or not. In the first case, flow reversal in the predominantly inviscid small-defect region that reaches close to the wall requires a pressure rise $O(1)$ acting on a relatively short streamwise distance, which contradicts the original assumption; in the second case, the transcendentally thin viscous sublayer prevents the formation of viscous-inviscid boundary layer interaction that is sufficiently strong to ensure a smooth continuation of the incident boundary layer into a separated shear layer (cf. Sykes 1980). In order to overcome this dilemma, two disparate routes to separation can be established: in the first situation, the assumption of a large velocity defect leads to a multi-layered flow structure that distinctly differs from that outlined here and in turn to the theory of turbulent marginal separation (Scheichl \& Kluwick 2007a,b). The massively separating flow considered here pertains to the second case. Then, the structure of the stagnant-flow near the leading edge of a bluff body provides the remedy as the turbulence-intensity level in the boundary layer further downstream seems inevitably associated with socalled slightly underdeveloped turbulence: the von Kármán number varies essentially algebraically with $\delta$, but the velocity defect is $O(1 / \ln R e)$, as in the classical theory. In contrast to what is known from a marginally separating turbulent boundary layer, here the logarithmic law of the wall is not eradicated as the vast bulk of the small-defect layer is transferred unchanged into a separated shear layer - a probably surprising observation.

As an intriguing interpretation of the present flow description, the boundary layer undergoing separation is always influenced by its laminar origins near the leading edge, irrespective of how large $R e$ is. Above all, this conclusion is interestingly supported by experimental findings (Schewe 2001), although yet not conclusively. The rapid evolution towards a free fully developed turbulent shear layer of finite width is likely to take place at a rather short distance downstream of separation (cf. Sychev 2010). The related mechanism is still to be ascertained, and much more effort both experimentally and in DNS is required in order to fully corroborate this flow picture.

It is noteworthy that the scenario of an underdeveloped turbulent boundary layer allows a self-consistent time-averaged picture of a quite rapid laminar-turbulent transition, and thus a complete description of the boundary layer from transition towards separation. Therefore, it is promising to complete the theory of the turbulent flow past a sharp trailing edge at angle of attack (cf. Melnik \& Chow 1975), associated with a Brillouin-Villat-type singularity in the potential flow. Beyond that, it might be useful for future self-consistent descriptions of, for example, turbulent boundary layer flows over wall-mounted obstacles (cf. Sykes 1980) or turbulent boundary layer/shock wave interaction (cf. Agrawal \& Messiter 1984 and references therein) that include separation. 
Apart from the description of the separated shear layer, open points mainly relate to the precise position $x=x_{\mathscr{D}}$ of inviscid flow detachment or, equivalently, the value of $k$. In lieu of attempting to settle this question first, here we have drawn attention to the local structure of the separating flow by having in mind a particular value of $k$. The latter is predicted for a prescribed body geometry by the separation criterion derived rationally from the interplay of the inner with the outer interaction mechanism, which is encouraging. The exploration of this criterion, based on a thorough investigation of the underlying vortex-flow problem, will prove crucial for further progress. The forecast of $x_{\mathscr{D}}$ is linked to the correct choice of the inviscid-flow model and in turn to solving the long-standing problem of the asymptotic structure of the largescale turbulent separated flow. This consists of the free shear layer along the free streamline $\mathscr{S}$ having a thickness that grows linearly with the arclength along $\mathscr{S}$, the adjacent near wake that exhibits (two) reversed-flow eddies, and the wake further downstream that results from the merging of the two former flow regions. In view of present knowledge, the chances of establishing a complete description of self-induced massive separation are viewed as high. When it comes to the investigation of viscous effects on the separated inviscid flow, we might expect an interesting comparison between the asymptotic eddy models of laminar steady flow (finally resolved in all essentials by Chernyshenko 1988, cf. Smith 1986; Sychev et al. 1998) and the turbulent case, partly due to the small-defect structure of the separating turbulent boundary layer (cf. Sychev 2010). This, among other things, is a topic of current and future exciting research.

Fruitful discussions with Professor Sergei I. Chernyshenko are gratefully acknowledged. The authors also thank the referees for helpful comments.

\section{Appendix A. The Brillouin-Villat singularity}

We adopt the well-known vorticity transport theorem, deduced from (2.2), and cast the problem governing the inviscid flow into the form

$$
\begin{gathered}
\nabla^{2} \psi_{0}=-\omega_{0}\left(\psi_{0} ; k\right), \quad y \geqslant y_{\mathscr{S}}(s ; k): \omega_{0}=0, \\
\psi_{0}(x, 0 ; k)=\psi_{0}\left(x, y_{\mathscr{S}}(s ; k) ; k\right)=0 .
\end{gathered}
$$

The kinematic boundary condition (A $1 b)$ reflects $(2.3)$ for $s \leqslant 0$. The quantity $\omega_{0}$ may exhibit a discontinuity at $y=y_{\mathscr{S}}$ for $s>0$. We have

$$
U_{\mathscr{S}}=\sqrt{u_{0}^{2}+v_{0}^{2}} \text { for } y=y_{\mathscr{S}}
$$

so that $U_{\mathscr{S}}=u_{0}$ for $s \leqslant 0$, according to (A 2) and (A $\left.1 b\right)$. This gives (3.3), which is in line with (A $1 a)$ and $(2.2 c)$. Then, the requirement for smooth flow detachment reads as

$$
s \rightarrow 0_{-}: \quad U_{\mathscr{S}} \rightarrow u_{0 \mathscr{D}}(k)>0, \quad s \rightarrow 0_{+}: \quad U_{\mathscr{S}_{+}} \rightarrow u_{0 \mathscr{D}}(k), \quad U_{\mathscr{S}_{-}} \rightarrow 0 .
$$

Moreover, our concern is the separation on the upper part of the cylinder, so that $\psi_{0} \geqslant 0$ for $y \geqslant y_{\mathscr{S}}$, whereas the stagnant-flow or backflow region under consideration is characterised by $\psi_{0}=\omega_{0}=0$ or both $\psi \leqslant 0$ and $\omega_{0}<0$ for $0 \leqslant y<y_{\mathscr{S}}$, respectively.

The pressure $p_{0}$ is required to be continuous on $\mathscr{S}$ since the asymptotically slender separated viscous shear layer along $\mathscr{S}$ emerging in the case (1.1) exhibits a correspondingly weak variation of $p$ in the direction transverse to $\mathscr{S}$ (which mostly compensates for centripetal forces proportional to $U_{\mathscr{S}}^{2}$ times the curvature of $\mathscr{S}$ ). 
Employing Bernoulli's theorem together with (2.4) then states that

$$
p_{0}+\frac{u_{0}^{2}+v_{0}^{2}}{2}=B_{0}\left(\psi_{0} ; k\right)= \begin{cases}1 / 2, & \psi_{0} \geqslant 0, \\ p_{0 \mathscr{D}}(k)-\int_{0}^{\psi_{0}} \omega_{0}(t ; k) \mathrm{d} t, & \psi_{0}<0,\end{cases}
$$

which yields (3.4) along with the relationship

$$
p_{\mathscr{S}}+U_{\mathscr{S}-}^{2} / 2=\left(1-u_{0 \mathscr{D}}^{2}\right) / 2, \quad \psi_{0}<0,
$$

calculated from (A 3). Finally, (A 1) is supplemented with the resulting dynamic jump condition

$$
U_{\mathscr{S}+}^{2}-U_{\mathscr{S}_{-}}^{2}=u_{0 \mathscr{D}}^{2},
$$

according to the jump of $B_{0}$ across $\mathscr{S}$, given by $1 / 2-p_{0 \mathscr{D}}$.

We now derive the final expansions (3.6)-(3.9) in a stepwise fashion by invoking (A 1) and (A 6).

(a) We advantageously introduce polar coordinates, see (3.5), and investigate (A 1) for $y \geqslant y_{\mathscr{S}}$ near $\mathscr{D}$. To this end, we express $\nabla^{2}$ in terms of $r, \theta$ by inspection of $(2.2 c)$ and (3.5). Since $\psi_{0} \sim u_{0 \mathscr{D}} y+\cdots, y=r \sin \theta$, as $r \rightarrow 0$, according to (3.3) and (A 3), in this limit one can write

$$
\begin{gathered}
\nabla^{2} \psi_{0} \sim\left[\nabla_{p}^{2}+\varkappa_{\mathscr{D}} \partial_{y}+\cdots\right][1+O(r)] \psi_{0}, \\
\nabla_{p}^{2}=r^{-1} \partial_{r}\left(r \partial_{r}\right)+r^{-2} \partial_{\theta}^{2}, \quad \partial_{y}=\sin \theta \partial_{r}+r^{-1} \cos \theta \partial_{\theta},
\end{gathered}
$$

with $\nabla_{p}^{2}$ being the Laplacian in the case of a planar surface $(\varkappa \equiv 0)$. In the present context, $\psi_{0}=0$ for $\theta=\pi$. The analysis of the eigensolutions of Laplace's equation $\nabla^{2} \psi_{0}=0$ then yields the asymptotic expansion

$$
\begin{gathered}
\psi_{0} / u_{0 \mathscr{D}} \sim r \sin \theta+\psi_{0 \lambda}(r, \theta ; k)+\cdots+\psi_{02}(r, \theta ; k)+\cdots, \quad \pi \geqslant \theta>0, \quad r \rightarrow 0,(\mathrm{~A} 8 a) \\
\psi_{0 \lambda}=a_{\lambda}(k) r^{\lambda} \sin [\lambda(\pi-\theta)], \quad \psi_{02}=r^{2}\left\{a_{2}(k) \sin (2 \theta)-\left(\varkappa_{\mathscr{D}} / 4\right)[1-\cos (2 \theta)]\right\}, \quad(\mathrm{A} 8 b)
\end{gathered}
$$

matching (3.3) as $\theta \rightarrow \pi$, with $\nabla_{p}^{2}\left[\psi_{0 \lambda}, \psi_{02}\right]=\left[0,-\varkappa_{\mathscr{D}}\right]$ and both the constant $\lambda>1$ and the dependences of the parameters $a_{\lambda} \neq 0$ and $a_{2}$ on $k$ unknown at this stage. Specifically, we seek the smallest value of $\lambda$ by utilising the kinematic and dynamic boundary conditions (A $1 b$ ) and (A 6), respectively, that hold on $\mathscr{S}$. (We notice the passive nature of the inherent breakdown of the expansion (A 8) in the limit $y-y_{\mathscr{S}} \rightarrow 0_{+}$. Hence, first we can ignore the region of non-uniformity, and the expansion of $\psi_{0}$ is restated solely by rearranging the terms that result from expanding each term in (A $8 a)$ in this limit.)

(b) Inserting (A 1b) into (A 8a) provides the one-term estimate $\theta \sim c_{\lambda} r^{\lambda-1}+\cdots$ and, by using (3.5), $\theta \sim c_{\lambda} s^{\lambda-1}+\cdots$ for $y_{\mathscr{S}} \sim c_{\lambda} s^{\lambda}+\cdots$, $c_{\lambda}=-a_{\lambda} \sin (\lambda \pi)>0$, as $s \rightarrow 0_{+}$. Consequently, we infer from (A 2) and (A 7) that $U_{\mathscr{S}_{+}}^{2} \sim\left[\left(\partial_{r} \psi_{0}\right)^{2}+r^{-2}\left(\partial_{\theta} \psi_{0}\right)^{2}\right]\left[1+O\left(\varkappa_{\mathscr{D}} r^{\lambda}\right)\right]$ for $y=y_{\mathscr{S}}$ in this limit. After some algebra, we derive from the aforementioned one-term approximation and the three-term expansion (A 8) the expression

$$
\begin{aligned}
\left(U_{\mathscr{S}+} / u_{0 \mathscr{D}}\right)^{2} \sim 1-2 \lambda a_{\lambda} s^{\lambda-1} \cos (\lambda \pi)+[\lambda & \left.+2(\lambda-1) \sin (\lambda \pi)^{2}\right] \lambda a_{\lambda}^{2} s^{2 \lambda-2} \\
& +4 a_{2} s+O\left(s^{\lambda}, s^{2}\right), \quad s \rightarrow 0_{+} .
\end{aligned}
$$

(c) Next, we envisage the flow in the slipstream zone close to $\mathscr{D}$, near which it is of a cusp-type shape, according to the estimate for $y_{\mathscr{S}}$ given in item (ii). In turn, for $\omega_{0}<0$, we have $\left|v_{0}\right| \ll\left|u_{0}\right|=O\left(U_{\mathscr{S}_{-}}\right)$in that flow region. One then finds 
that $\psi_{0}$ assumes the self-similar form $\psi_{0} /\left(y_{\mathscr{S}} U_{\mathscr{S}_{-}}\right) \sim f(\xi)+\cdots, \xi=y / y_{\mathscr{S}}$, as $s \rightarrow 0_{+}$. Substituting this expression into (A1) results in the least-degenerate form of this problem,

$$
\begin{gathered}
U_{\mathscr{S}_{-}} f^{\prime \prime}-2 \lambda c_{\lambda}^{2} s^{2 \lambda-1} U_{\mathscr{S}_{-}}^{\prime}\left(\xi f^{\prime}-f\right)+c_{\lambda}^{2} s^{2 \lambda} U_{\mathscr{S}-}^{\prime \prime} f \sim-c_{\lambda} s^{\lambda} \omega_{0}, \quad s \rightarrow 0_{+}, \\
f(0)=f(1)=0, \quad f^{\prime}(1)=1,
\end{gathered}
$$

where primes on $U_{\mathscr{S}_{-}}$denote derivatives with respect to $s$. The relationships (A 10) give rise to a boundary-value problem fixing both $f(\xi)$ and $U_{\mathscr{S}_{-}}$near $\mathscr{D}$. The first term in (A 10a) contains the highest derivative with respect to $\xi$ and must, therefore, be retained to leading order. Since $U_{\mathscr{S}_{-}} \rightarrow 0_{+}$, according to (A 6), the second and thus the third term in (A 10a) are negligibly small. Hence, (A 10a) is seen to reduce to a balance of the first term and that on the right-hand side, where we impose the apparent restriction that $\omega_{0}$ is bounded as $\psi_{0} \rightarrow 0_{-}$and define $\omega_{0 \mathscr{D}}=\omega_{0}\left(0_{-} ; k\right) \leqslant 0$. By using (A 10b), we arrive at the reverse-flow representation $f(\xi)=\left(\xi^{2}-\xi\right) / 2$, as the possibly slowest decay of $U_{\mathscr{S}_{-}}$reads as

$$
U_{\mathscr{S}_{-}} \sim-\omega_{0 \mathscr{D}} c_{\lambda} s^{\lambda}+\cdots, \quad s \rightarrow 0_{+} .
$$

(d) We now evaluate (A 6) by inserting (A9) and (A 11), in order to fix $\lambda, a_{\lambda}$, $a_{2}$. One then finds that $U_{\mathscr{S}_{+}}=o\left(s^{\lambda-1}\right)$. From (A 9), we infer that $\lambda$ is a member of the sequence of eigenvalues $\lambda=3 / 2,5 / 2,7 / 2, \ldots$, as $\psi_{0 \lambda}$ is seen to represent a homogeneous eigenfunction of the Laplacian with $\partial_{\theta} \psi_{02}=0$ for $\theta=0$ and $\psi_{02}=0$ for $\theta=\pi$ and coefficients $a_{\lambda}$ that cannot be determined by the local analysis. Next, we conclude that integer powers of $s$ have to be eliminated in (A9), which demands $a_{2}=-15 a_{\lambda}^{2} / 16$ for $\lambda=3 / 2$ and $a_{2}=0$ for $\lambda>3 / 2$. Also, higher-order terms indicated by dots are seen to be $O\left(r^{\lambda+1}\right)$ in (A $\left.8 a\right)$ and $O\left(r^{\lambda}\right), O\left(s^{\lambda}\right)$ and $O\left(s^{\lambda+1}\right)$, respectively, in the three relationships expressing the shape of $\mathscr{S}$ as $s \rightarrow 0_{+}$in item (ii) above. However, only the terms stated explicitly will turn out to significantly affect the further description of separation and are thus under focus here.

(e) Let $\lambda$ take on its minimum value $3 / 2$, corresponding to the most general application. We then define $a_{3 / 2}=a_{\lambda}=c_{\lambda}, \psi_{0,3 / 2}=\psi_{0 \lambda}$, where it proves convenient to introduce the constant $k$ by setting $a_{3 / 2}=4 k / 3$. Eventually, we restate (A 8 ) in the form (3.6) and obtain the expressions (3.7b) and (3.9a) from (A 6) and (A 11), respectively, where (3.5) and (A 7) are employed. We note that (3.6)-(3.9) capture the general case $\lambda \geqslant 3 / 2$ as $\lambda=3 / 2$ for $k>0$ and the case $\lambda=5 / 2$ in the degenerate limit $k=0$.

\section{Appendix B. Computation of potential flow}

The classical way to solve the problem of potential flow around a finite twodimensional body that exhibits (two) detaching streamlines confining a dead-water cavity, as formulated by (A 1$)$ with $\omega_{0} \equiv 0$, is provided by the Levi-Cività method (with certain additions, cf. Gurevich 1979).

\section{B.1. Preliminaries}

The associated conformal transformation maps the region of flow in the physical plane (see figure 1), with complex variable $z$, onto a closed contour lying in the plane of a complex variable $\zeta$ and its interior (see figure 8). This contour consists of the upper half-unit circle $\mathscr{H}^{\prime}$, the points $\zeta=\mp 1$, and the portion $\mathscr{R}^{\prime}$ of the real axis between these points, which are, respectively, the images of the wetted fraction of the body surface (not adjoining the fluid at rest), the points of flow detachments and the 


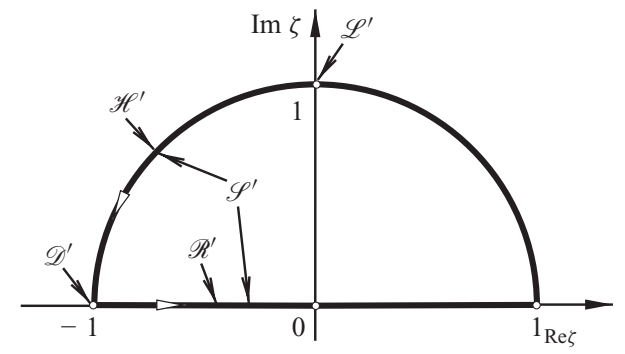

FIGURE 8. Flow situation in the complex $\zeta$-plane (for labelling see $\S$ B.1).

detached parts of the free streamlines. Here, the bijective function $\zeta(z)$ is regarded as analytic in the whole $z$-plane except for $z=\infty$, having the image $\zeta=0$, where it has a pole. For a symmetric problem as considered here, let $\mathscr{D}^{\prime}$ at $z=-1$ and $\mathscr{L}^{\prime}$ at $\zeta=\mathrm{i}$ be the images of the detachment and stagnation points $\mathscr{D}$ and $\mathscr{L}$, respectively. Accordingly, the upper detaching streamline $\mathscr{S}$ is mapped onto the curve $\mathscr{S}^{\prime}$, which consists of the right halves of $\mathscr{H}^{\prime}$ and $\mathscr{R}^{\prime}$.

Also, we introduce the complex potential $w_{0}(z ; k)=\varphi_{0}+\mathrm{i} \psi_{0}$ and the function $\Omega(\zeta ; k)=\mathrm{i} \ln \left[\left(\mathrm{d} w_{0} / \mathrm{d} z\right) / u_{0 \mathscr{D}}\right]=\vartheta_{0}+\mathrm{i} \ln \left(V / u_{0 \mathscr{D}}\right)$. We stress that $w_{0}$ and $\Omega$ are analytic inside and on the contour comprising $\mathscr{H}^{\prime}$ and $\mathscr{R}^{\prime}$ apart from, respectively, $\zeta=0$ (where $w_{0}$ has a pole) and $\zeta=\mathrm{i}$ as

$$
\Omega \sim \mathrm{i} \ln (\zeta-\mathrm{i})+O(1), \quad \zeta-\mathrm{i} \rightarrow 0
$$

(since $\mathrm{d} w_{0} / \mathrm{d} z$ vanishes in a regular manner at the point $\mathscr{L}$ ). Thus, $V$ and $\vartheta_{0}$ denote the (positive) flow speed and the flow angle, respectively. On $\mathscr{H}^{\prime}$, we have $\psi_{0}=0$ and $\vartheta_{0}=\phi$, with $\phi$ satisfying (2.1). The image of $\mathscr{S}$ is then parametrised in terms of a real parameter $\varrho$ that increases in the flow direction (as indicated by the arrows on $\mathscr{S}^{\prime}$ and $\mathscr{R}^{\prime}$ in figure 8 ) by setting, respectively,

$$
\begin{array}{rll}
\zeta=\exp [\mathrm{i}(\pi+\varrho)], \quad-\pi / 2 \leqslant \varrho \leqslant 0: & \psi_{0}=0, \quad V=U_{\mathscr{S},} \quad \vartheta_{0}=\phi, \\
\zeta=-1+\varrho, \quad 0<\varrho<1: & \psi_{0}=0, \quad V=U_{\mathscr{S}_{+}} \equiv u_{0 \mathscr{D}}(>0) .
\end{array}
$$

The last identity follows by equating $p_{\mathscr{S}}$ and $U_{\mathscr{S}_{-}}$(A 5) with the constant cavity pressure $\left(1-u_{0 \mathscr{D}}^{2}\right) / 2 p_{0}$ and 0 , respectively, according to (A 3$)$, and upon substitution in (A 6). Finally, the corresponding relationships

$$
\begin{array}{rll}
s \leqslant 0, \quad y=0: & \mathrm{d} \varphi_{0} / \mathrm{d} l=U_{\mathscr{S}}(x ; k), \quad l=s, \quad x=x_{\mathscr{D}}+s, \\
s>0, \quad y=y_{\mathscr{S}}(s ; k): & \mathrm{d} \varphi_{0} / \mathrm{d} l=u_{0 \mathscr{D}}
\end{array}
$$

between the real flow potential $\varphi_{0}$ and $V$ holding on $\mathscr{S}$ are imposed, so that $l$ denotes the arclength along $\mathscr{S}$ measured from $\mathscr{D}$. Also, $\varphi_{0}>0$ and $\mathrm{d} \varphi_{0} / \mathrm{d} \varrho>0$ for $-\pi / 2 \leqslant \varrho<1$.

\section{B.2. Flow along $\mathscr{S}$ close to detachment}

The results $(3.7 b)$ and $(3.9 b)$ evince the behaviour of the potential flow near the B-V singularity with sufficient accuracy. We first demonstrate in a nutshell how these are recovered readily by exploiting the real parts of the Taylor series about $\mathscr{D}^{\prime}$ derived from (B 2),

$$
\begin{aligned}
w_{0} & \sim \varphi_{\mathscr{D}}+W_{2}\left[(\zeta+1)^{2}+(\zeta+1)^{3}\right]+O\left[(\zeta+1)^{4}\right], \\
\Omega & \sim \phi_{\mathscr{D}}+\Omega_{1}(\zeta+1)+\Omega_{2}(\zeta+1)^{2}+O\left[(\zeta+1)^{3}\right],
\end{aligned}
$$


with some real coefficients $\varphi_{\mathscr{D}}(>0), W_{2}(>0), \phi_{\mathscr{D}}, \Omega_{1}, \Omega_{2}$. By substituting (B $\left.2 a\right)$ into (B 4), the image of the flow on $\mathscr{S}$ near $\mathscr{D}$ for $s \rightarrow 0_{-}$is then represented as

$$
\begin{aligned}
\varphi_{0} & \sim \varphi_{\mathscr{D}}-W_{2} \varrho^{2}+O\left(\varrho^{4}\right), \\
U_{\mathscr{S}} / u_{0 \mathscr{D}} & \sim \exp \left[\Omega_{1} \varrho+O\left(\varrho^{3}\right)\right] \sim 1+\Omega_{1}\left(\varrho+\varrho^{2} / 2\right)+O\left(\varrho^{3}\right), \\
\phi & \sim \phi_{\mathscr{D}}+\left(\Omega_{1} / 2-\Omega_{2}\right) \varrho^{2}+O\left(\varrho^{4}\right),
\end{aligned}
$$

as $\varrho \rightarrow 0_{-}$. By rewriting $(\mathrm{B} 3 a)$ as $\left(\mathrm{d} \varphi_{0} / \mathrm{d} \varrho\right)(\mathrm{d} \varrho / \mathrm{d} s)=U_{\mathscr{S}}$, we now extract from (B $\left.5 a\right)$ the relationship $\mathrm{d}\left(\varrho^{2}\right) / \mathrm{d} s \sim-\left(u_{0 \mathscr{D}} / W_{2}\right)\left[1+\Omega_{1} \varrho+O\left(\varrho^{2}\right)\right]$, as $s \rightarrow 0_{-}$. One then easily obtains

$$
\begin{gathered}
\Omega_{1} \varrho \sim 2 k(-s)^{1 / 2}\left[1+2 k(-s)^{1 / 2} / 3+O(-s)\right], \quad s \rightarrow 0_{-}, \\
k=\Omega_{1} \varsigma^{1 / 2} / 2, \quad \varsigma=u_{0 \mathscr{D}} / W_{2}(>0) .
\end{gathered}
$$

Here we have reintroduced the potential flow parameter $k$. Inserting (B 6) into (B 5b) finally yields the three-term expansion (3.7b). Furthermore, we infer from (B 5c) that

$$
\varsigma\left(\Omega_{1} / 2-\Omega_{2}\right)=x_{\mathscr{D}} .
$$

For the flow along the detached portion of $\mathscr{S}$ governed by (B $2 b)$ and (B $3 b)$, the relations (B 4) then give $\varrho \sim(\varsigma l)^{1 / 2}-(\varsigma l) / 2+O\left(l^{3 / 2}\right)$ and, in turn,

$$
\vartheta_{0} \sim \phi_{\mathscr{D}}+2 k l^{1 / 2}-x_{\mathscr{D}} l+O\left(l^{3 / 2}\right), \quad l \rightarrow 0_{+} .
$$

This last result accounts for the curvature $-\mathrm{d} \vartheta_{0} / \mathrm{d} l$ of $\mathscr{S}$ (with the sign conforming to the definition $(2.1)$ of $\chi$ ) immediately downstream of $\mathscr{D}$, in agreement with $(3.9 b)$ since $l \sim s+\cdots, \mathrm{d} \vartheta_{0} / \mathrm{d} l \sim \mathrm{d}^{2} y_{\mathscr{S}} / \mathrm{d} s^{2}+\cdots$, as $s \rightarrow 0_{+}$. In this context, the B-V singularity is commonly described by (3.8) and (B 9), see e.g. Sychev et al. (1998, p. 10).

We have shown that the representation of the flow in the $\zeta$-plane, together with the choice of the auxiliary parameter $\varrho$ as the independent variable, allows a convenient regularisation of the B-V singularity. Nonetheless, calculating the local form (3.6) of $\psi_{0}$ by means of the method described in $\S$ B.2 is definitely less gratifying when compared to the procedure outlined in $\S 3.3$. On the other hand, the Levi-Cività method provides a powerful tool for constructing a numerical solution of the overall potential flow problem (A 1).

\section{B.3. Global flow: numerical treatment}

Here, we restrict the application of the Levi-Cività method to the case of an open cavity, i.e. by anticipating $u_{0 \mathscr{D}}=1$ in (B $2 b$ ) by (A 4 ) and (2.4). The main focus lies on the determination of the surface flow speed $U_{\mathscr{S}}(x ; k)$ for a prescribed (non-negative) value of $k$.

We consider a specific conformal mapping $\zeta(z)$ that achieves

$$
w_{0}=[\varpi(k)(\zeta+1 / \zeta) / 2]^{2},
$$

with the yet unknown function $\varpi(k)$ being real. The relation (B 10) has all the desired properties of the potential $w_{0}$ discussed in $\S$ B.1, also see (B 2). In addition, it reflects the symmetry of the problem as $\phi_{0}$ and $\psi_{0}$ are respectively symmetric and antisymmetric with respect to both the imaginary and the real axes of the $\zeta$-plane. Corresponding requirements then have to be met by $\Omega=\vartheta_{0}+\mathrm{i} \ln V$ : in the upper half-plane, $\vartheta_{0}(V)$ are antisymmetric (symmetric) with respect to the imaginary axis, and the converse conditions hold in the lower half-plane; note (B $2 b)$ with $V=1$ and $\vartheta_{0}=0$ for $z=\infty$ or $\zeta=0$ ). Moreover, (B 1) gives evidence that

$$
\Xi(\zeta ; k)=\Omega(\zeta ; k)-\pi-\mathrm{i} \ln [(\zeta-\mathrm{i}) /(\zeta+\mathrm{i})]
$$


is analytic inside and on the boundary of the unit circle and exhibits symmetry properties identical with those of $\Omega$. That is, $\Xi$ can be expanded in the Taylor series $\Xi \sim \sum_{n=1}^{\infty} \Xi_{2 n-1} \zeta^{2 n-1},|\zeta| \leqslant 1$, with $\operatorname{Im} \Xi_{n}=0$.

The aforementioned symmetry properties are revealed by expansions of the form $\phi \sim \pi / 2+O(v), \quad U_{\mathscr{S}} / U_{\mathscr{S}_{0}}(k) \sim v+U_{1}(k) v^{3}+O\left(v^{5}\right), \quad$ as $\quad v=\pi / 2+\varrho \rightarrow 0_{+}$, with $U_{\mathscr{S}_{0}}(k)$ being a positive quantity. They confirm the stagnating-flow behaviour (see figures $1 a$ and 8). By noticing (B $2 a$ ), we then end up with the Fourier representation of $\Xi\left[\mathrm{e}^{\mathrm{i}(\pi+\varrho)} ; k\right]$,

$$
\begin{gathered}
\varrho=-\frac{\pi}{2}: \quad \phi=\frac{\pi}{2}, \Upsilon=\ln \left(2 U_{\mathscr{S} 0}\right)=-\sum_{n=1}^{\infty}(-1)^{n} \Xi_{2 n-1}, \\
0 \geqslant \varrho>-\frac{\pi}{2}: \quad \phi-\frac{\pi}{2}+\mathrm{i} \ln \frac{U_{\mathscr{S}}(1-\sin \varrho)}{\cos \varrho}=-\sum_{n=1}^{\infty} \Xi_{2 n-1} \exp [\mathrm{i}(2 n-1) \varrho] .
\end{gathered}
$$

In turn, the antisymmetry of $U_{\mathscr{S}}$ with respect to $v=0$ is confirmed by the limiting form $\operatorname{Im} \Xi\left[\mathrm{e}^{\mathrm{i}(\pi / 2+v)} ; k\right] \sim \Upsilon+v^{2}\left(U_{1}-1 / 12\right)+O\left(v^{4}\right)$, as $v \rightarrow 0_{+}$. Also, the evaluation of (B 2a), (B 11) and (B 12b) for $\varrho=0$ gives

$$
\phi_{\mathscr{D}}=\Omega_{0}=\pi / 2-\sum_{n=1}^{\infty} \Xi_{2 n-1}
$$

A collocation method can be set up for a prescribed inclination angle (flow angle) $\phi(x), 0 \leqslant x \leqslant x_{\mathscr{D}}$, of the body surface to approximately compute $\varpi$ and $\Xi_{n}$, with $n=1,2, \ldots, N$ for some index $N$. First, we notice that (B 10) yields $\varphi_{0}=(\varpi \cos \varrho)^{2}$ on $|\zeta|=1$ and find $\varpi=W_{2}^{1 / 2}$ by using (B 5a). Then, we rewrite (B $3 a$ ) with the help of (2.1),

$$
\begin{aligned}
-\pi / 2 \leqslant \varrho<0: & \varkappa \varpi^{2} \sin (2 \varrho)=U_{\mathscr{S}} \mathrm{d} \phi / \mathrm{d} \varrho, \\
\varrho=0: & 2 \varkappa_{\mathscr{D}} \varpi^{2}=\mathrm{d}^{2} \phi / \mathrm{d} \varrho^{2} ;
\end{aligned}
$$

applying de l'Hôpital's rule to (B 14a) gives (B 14b), in agreement with (B 5c), (B 7) and (B 8). In turn, the elimination of $\Omega_{1}$ from (B 7) with the aid of (B $5 b$ ) reveals the effect of the control parameter $k$ in terms of a compatibility condition

$$
\varrho=0: \quad 2 k \varpi=\mathrm{d} U_{\mathscr{S}} / \mathrm{d} \varrho .
$$

We now treat the surface curvature $x$ as a function of $\phi$ and have the right-hand sides of (B 14) and (B 15) expressed through (B 12), where we terminate the sum at some index $n=N, N$ being sufficiently large. Then, the evaluation of (B 14a) at $N-1$ different locations $\varrho=\rho_{n},-\pi / 2<\varrho_{n}<0, n=1,2, \ldots, N-1$, supplemented with (B 14b) and (B 15), establishes a set of $N+1$ nonlinear transcendental equations for $N$ unknown coefficients $\Xi_{2 n-1}, n=1,2, \ldots, N$, and $\varpi(>0)$. Their roots can be found efficiently by using standard numerical methods. Finally, (B 13) gives the flow angle $\phi=\phi_{\mathscr{D}}(k)$ at the detachment point $\mathscr{D}$. As the simplest but most important example, we consider the canonical problem of the flow around the unit cylinder, where $x \equiv 1, x=\pi-\phi, x_{\mathscr{D}}(k)=\pi-\phi_{\mathscr{D}}(k)$ (see figure $\left.1 a\right)$. Here we choose equidistantly spaced values of $\varrho_{n}=(\pi / 2)(n / N-1), n=1,2, \ldots, N$, and $N=350$. It is demonstrated in the theory of Fourier analysis that $\Xi_{N}=O\left(N^{-2}\right)$ or smaller for $N \gg 1$. However, here even $\Xi_{N}=o\left(N^{q}\right)$ for any $q<0$ due to analyticity of $\Xi$, which, in turn, suggests the absolute numerical error then to be reduced to the order of $10^{-5}$ or smaller.

The physically admissible solutions have been discussed extensively by Scheichl et al. (2008) and Scheichl \& Kluwick (2008b) for the particular range $0 \leqslant k \leqslant k_{c} \doteq 0.4911$ (referring to monotonically increasing values of the separation angle $x_{\mathscr{D}}$ ). Specifically, the limiting values attract most interest. First, $x_{\mathscr{D}}(0) \doteq 55^{\circ} 2^{\prime} 30^{\prime \prime}$ denotes the so-called 
B-V angle that points to laminar separation (Sychev 1972; Sychev et al. 1998; Smith 1977). A simplified version of the procedure presented here, successfully applied to this case much earlier, must be attributed to several authors appreciated by Gurevich (1979). Second, let us refer to figure $2(a)$ discussed in $\S 3.2$ : then $x_{\mathscr{D}}\left(k_{c}\right) \doteq 124^{\circ} 12^{\prime} 11^{\prime \prime}$ represents the critical downstream limit of $x_{\mathscr{D}}$ in the sense of case II. Note that values of $k$ larger than $k_{c}$ imply a contradiction to the original assumption of a semi-infinite cavity as they predict a non-smooth intersection of $\mathscr{S}$ with the real (symmetry) axis of the $z$-plane at a finitely remote point. A simple criterion for the occurrence of this situation is indicated by the Taylor expansion of (B 11) about $\zeta=0$. This gives $\vartheta_{0} \sim \pi-\left(2-\Xi_{1}\right) \zeta+\left(2 / 3+\Xi_{2}\right) \zeta^{3}+O\left(\zeta^{5}\right)$ and thus describes the shape of $\mathscr{S}$ far downstream when $\zeta \rightarrow 0_{-}$. It asymptotes to the well-known Kirchhoff parabola (case I, $0 \leqslant k<k_{c}$ ) for $\Xi_{1}<2$, which degenerates to a cusp (case II, $k=k_{c}$ ) for $\Xi_{1}=\Xi_{1 c}=2$.

Early numerical computations of $\mathrm{H}-\mathrm{K}$ flows around the circular cylinder were carried out by Woods (1955). The integral method exploited by him, however, is considerably more complicated and less straightforward to apply compared with that proposed here, based on the Levi-Cività method.

\section{REFERENCES}

Agrawal, S. \& Messiter, A. F. 1984 Turbulent boundary-layer interaction with a shock wave at a compression corner. J. Fluid Mech. 143, 23-46.

BATCHELOR, G. K. 1956a On steady laminar flow with closed streamlines at large Reynolds number. J. Fluid Mech. 1 (2), 177-190.

BATCHELOR, G. K. 1956b A proposal concerning laminar wakes behind bluff bodies at large Reynolds number. J. Fluid Mech. 1 (4), 388-398.

Brown, S. N. \& Stewartson, K. 1970 Trailing-edge stall. J. Fluid Mech. 42 (3), 561-584.

Chernyshenko, S. I. 1988 The asymptotic form of the stationary separated circumfluence of a body at high Reynolds numbers. PMM U.S.S.R. 52 (6), 746-753, original Russian article in Prikl. Mathem. Mekhan. 52 (6), 1988, 958-966.

Diesperov, V. N. 1984 On the existence and uniqueness of self-similar solutions describing the flow in mixing layers. Dokl. Akad. Nauk SSSR 275 (6), 1341-1346.

van Dommelen, L. L. \& Shen, S. F. 1984 Interactive separation from a fixed wall. In Numerical and Physical Aspects of Aerodynamic Flows II, Proc. 2nd Symp. California State University, Long Beach, CA, 17-20 January, 1983 (ed. T. Cebeci), pp. 393-402. Springer.

EPPLER, R. 1954 Beiträge zur theorie und Anwendung der Unstetigen Strömungen. Rat. Mech. Anal. 3, 591-644 (in German).

Gurevich, M. I. 1979 Theory of Jets in Ideal Fluids, 2nd edn. Academic Press (original Russian edition: 1965, Nauka).

Jones, G. W., Cincotta, J. \& Walker, W. 1969 Aerodynamic forces on a stationary and oscillating circular cylinder at high Reynolds numbers. NASA Tech. Rep. R-300.

KLEBANOFF, P. S. 1955 Characteristics of turbulence in a boundary layer with zero pressure gradient. NASA Tech. Rep. 1247; also NACA Tech. Note 3178 (1954).

KLuwick, A. \& SCHeichl, B. 2009 High-Reynolds-number asymptotics of turbulent boundary layers: from fully attached to marginally separated flows. In BAIL 2008 - Boundary and Interior Layers. Proceedings of the Intl Conf. Boundary and Internal Layers - Computational and Asymptotic Methods, Limerick, July 2008 (ed. A. F. Hegarty, N. Kopteva, E. O'Riordan \& M. Stynes), Lecture Notes in Computational Science and Engineering, vol. 69, pp. 3-22. Springer.

Kolmogorov, A. N. 1961 A refinement of previous hypotheses concerning the local structure of turbulence in a viscous incompressible fluid at high Reynolds number. J. Fluid Mech. 13 (1), $82-85$.

Korolev, G. L. 1980 Numerical solution of the asymptotic problem of laminar boundary-layer separation from a smooth surface. Uch. Zap. TsAGI 11 (2), 27-36.

MelNiK, R. E. 1989 An asymptotic theory of turbulent separation. Comput. Fluids 17 (1), 165-184. 
Melnik, R. E. \& Chow, R. 1975 Asymptotic theory of two-dimensional trailing-edge flow. NASA Tech. Rep. NAS1-12426.

Michel, R., QuÉmard, C. \& Durant, R. 1969 Hypothesis on the mixing length and application to the calculation of the turbulent boundary layers. In Proc. Computation of Turbulent Boundary Layers - 1968 AFOSR-IFP-Stanford Conference. Vol. I: Methods, Predictions, Evaluation and Flow Structure (ed. S. J. Kline, M. V. Morkovin, G. Sovran \& D. J. Cockrell), pp. 195-207. Stanford University.

Monin, A. S. \& Yaglom, A. M. 1971 Statistical Fluid Mechanics: Mechanics of Turbulence, vol. 1. MIT.

Nagib, H. M., Chauhan, K. A. \& Monkewitz, P. A. 2007 Approach to an asymptotic state for zero pressure gradient turbulent boundary layers. Phil. Trans. R. Soc. A 365 (1852), 755-770.

NeILAND, V. Y. 1971 Flow behind the boundary-layer separation point in a supersonic stream. Fluid Dyn. 6 (3), 378-384; original Russian article in Izv. Akad. Nauk SSSR, Mekh. Zhidk. $i$ Gaza (3), 1971, 19-27.

Neish, A. \& Sмiтh, F. T. 1988 The turbulent boundary layer and wake of an aligned flat plate. J. Engng Math. 22 (1), 15-42.

Neish, A. \& Smith, F. T. 1992 On turbulent separation in the flow past a bluff body. J. Fluid Mech. 241, 443-467.

RothmaYer, A. P. \& Smith, F. T. 1998 Part III. High Reynolds number asymptotic theories. In The Handbook of Fluid Dynamics (ed. R. W. Johnson), pp. III.1-25.26. Springer.

SandberG, R. D. \& Sandham, N. D. 2008 Direct numerical simulation of turbulent flow past a trailing edge and the associated noise generation. J. Fluid Mech. 596, 353-385.

SAndborn, V. A. \& LiU, C. Y. 1968 On turbulent boundary-layer separation. J. Fluid Mech. 32 (2), 293-304.

SCheichl, B. \& KLUwick, A. 2007a On turbulent marginal boundary layer separation: how the half-power law supersedes the logarithmic law of the wall. Intl J. Comput. Sci. Math. 1 (2-4), 343-359.

Scheichl, B. \& Kluwick, A. $2007 b$ Turbulent marginal separation and the turbulent Goldstein problem. AIAA J. 45 (1), 20-36.

SCHEICHL, B. \& Kluwick, A. 2008 a Asymptotic theory of bluff-body separation: a novel shearlayer scaling deduced from an investigation of the unsteady motion. J. Fluids Struct. 24 (8), 1326-1338. Special Issue on the IUTAM Symposium on Separated Flows and Their Control.

SCHEICHL, B. \& KLuwick, A. $2008 b$ Level of turbulence intensities associated with bluff-body separation for large values of the Reynolds number. AIAA Meeting Paper 2008-4348.

Scheichl, B. \& KLUwick, A. 2009 Evolution of a boundary layer from laminar stagnation-point flow towards turbulent separation. In Progress in Turbulence III. Proc. iTi Conference in Turbulence 2008 (ed. J. Peinke, M. Oberlack \& A. Talamelli), Springer Proceedings in Physics, vol. 131, pp. 187-190. Springer.

Scheichl, B., Kluwick, A. \& Alletto, M. 2008 'How turbulent' is the boundary layer separating from a bluff body for arbitrarily large Reynolds numbers? Acta Mech. 201 (1-4), 131-151.

SchewE, G. 2001 Reynolds-number effects in flows around more-or-less bluff bodies. J. Wind Engng Ind. Aerodyn. 89 (14), 1267-1289.

Schlichting, H. \& Gersten, K. 2003 Boundary-layer Theory, 8th edn. Springer.

SCHNEIDER, W. 1991 Boundary-layer theory of free turbulent shear flows. Z. Flugwiss. Weltraumforsch. (J. Flight Sci. Space Res.) 15 (3), 143-158.

Smith, F. T. 1977 The laminar separation of an incompressible fluid streaming past a smooth surface. Proc. R. Soc. Lond. A 356 (1687), 443-463.

SMith, F. T. 1979 Laminar flow of an incompressible fluid past a bluff body: the separation, reattachment, eddy properties and drag. J. Fluid Mech. 92 (1), 171-205.

Sмiтh, F. T. 1985 A structure for laminar flow past a bluff body at high Reynolds number. J. Fluid Mech. 155, 175-191.

Sмith, F. T. 1986 Concerning inviscid solutions for large-scale separated flows. J. Engng Math. 20 (3), 271-292.

Stewartson, K. \& Williams, P. G. 1973 On self-induced separation II. Mathematika 20 (6), 90-108.

Sychev, V. V. 1972 Laminar separation. Fluid Dyn. 7 (3), 407-417; original Russian article in Izv. Akad. Nauk SSSR, Mekh. Zhidk. i Gaza (3), 1972, 47-59. 
Sychev, V. V. 1983 Asymptotic theory of turbulent separation. Fluid Dyn. 18 (4), 532-538, original Russian article in Izv. Akad. Nauk SSSR, Mekh. Zhidk. i Gaza (4), 1983, 47-54.

Sychev, V. V. 1987 Theory of self-induced separation of a turbulent boundary layer. Fluid Dyn. 22 (3), 371-379, original Russian article in Izv. Akad. Nauk SSSR, Mekh. Zhidk. i Gaza (3), 1987, 51-60.

Sychev, V. V. 2010 On Asymptotic theory of separated flows past bodies. Fluid Dyn. 45 (3), 441-457, original Russian article in Izv. Ross. Akad. Nauk, Mekh. Zhidk. i Gaza 45 (3), 2010, 110-128.

Sychev, V. V., Ruban, A. I., Sychev, Vic. V. \& Korolev, G. L. 1998 Asymptotic Theory of Separated Flows. Cambridge University Press.

Sychev, V. V. \& Sychev, VIK. V. 1980 On turbulent separation. USSR Comput. Math. Phys. 20, $133-145$.

SYKES, R. I. 1980 An asymptotic theory of incompressible turbulent boundary-layer flow over a small hump. J. Fluid Mech. 101 (3), 631-646.

Woods, L. C. 1955 Two-dimensional flow of a compressible fluid past given curved obstacles with infinite wakes. Proc. R. Soc. Lond. A 227 (1170), 367-386.

Wu, T.. Y. 1972 Cavity and wake flows. Annu. Rev. Fluid Mech. 4, 242-284.

Zdravkovich, M. M. 1997 Flow Around Circular Cylinders: A Comprehensive Guide through Flow Phenomena, Experiments, Applications, Mathematical Models, and Computer Simulations. Vol. 1: Fundamentals. Oxford University Press. 